\title{
RESPONSE OF CORRUGATED INTERFACE ON INCIDENT QSV-WAVE IN MONOCLINIC ELASTIC HALF-SPACES
}

\author{
S.S. SINGH ${ }^{\star}$ \\ Department of Mathematics and Computer Science \\ Mizoram University, Aizawl -796 004 \\ Mizoram, INDIA \\ E-mail: saratcha32@yahoo.co.uk \\ J. LALVOHBIKA \\ Department of Mathematics, Pachhunga University College \\ Aizawl -796 001, Mizoram, INDIA \\ E-mail: vohbikajongte@gmail.com
}

\begin{abstract}
This paper is concerned with the problem of reflection and transmission of elastic waves due to an incident plane qSV-wave at a corrugated interface between two dissimilar monoclinic elastic half-spaces. Due to the corrugated nature of the interface, there exist regularly and irregularly reflected and transmitted elastic waves. Using Rayleigh's method of approximation, the reflection and transmission coefficients of regular and irregular waves are obtained for the first order of approximation. We have found that these coefficients are functions of the angle of incidence, elastic constants, corrugation and the frequency parameter. These coefficients are obtained for a special type of interface, $z=d \cos p y$. We have computed these coefficients for a particular model and discussed the effects of corrugation and frequency parameter.
\end{abstract}

Key words: qSV and qP-waves, reflection and transmission coefficients, monoclinic medium, corrugated interface, phase velocity.

\section{Introduction}

Elastic wave propagation is fascinating subject that deals with numerous problems in various fields such as seismology, geophysics, tele-communication (signal processing), medicine (echography), metallurgy (non-destructive testing) and earthquake engineering. These waves are useful in detection of notches and faults in different types of materials such as in railway tracks, buried land-mines, etc. The technique of seismic wave propagation is a tool for investigating the internal structure of the Earth and also used for exploration of valuable materials such as minerals, crystals, fluids (oils, water), etc. beneath the earth surface. When the seismic signal passes through different layers/discontinuities inside the Earth, the phenomena of reflection and transmission take place. These signals carry lots of information about the Earth structures see: Chattopadhyay and Choudhury [1], Chattopadhyay et al. [2], Dowaikh and Ogden [3], Sheriff and Geldart [4], Singh [5], Singh and Khurana [6], Udias [7] and others.

The propagation of elastic waves and their reflection/transmission from discontinuities/ interfaces are a great concern of many researchers. Chattopadhyay and Saha $[8,9]$ obtained the reflection and transmission coefficients of $P$ and qSV-waves at a plane interface between two different monoclinic media. Nayfeh [10] derived analytical expressions for the reflection and transmission coefficient from the interface of liquid/anisotropic half-spaces possessing monoclinic symmetry. Singh and Khurana [11] also investigated the reflection and transmission of $P$ and SV-waves at the interface between two monoclinic elastic half-spaces. But

\footnotetext{
${ }^{*}$ To whom correspondence should be addressed
} 
there is much experimental evidence that the discontinuities/interfaces between layers are not perfectly plane, but they are of irregular/corrugated nature. These irregular natures of the interfaces affect the reflection and transmission of elastic waves. Thus, it is important to take into account the problems related with the effects of irregular interfaces. It was Rayleigh [12] who first discussed the problems of reflection and transmission phenomena of light/sound waves from an irregular boundary surface. In his method, the function defining the corrugated interface in the expressions of boundary conditions is expanded in Fourier series and the unknown coefficients are determined upto any given order of approximation in terms of small parameter characteristics of magnitude and slope of corrugated interface. Later, Rayleigh's technique was applied to various other fields to study the reflection and transmission phenomena of waves at an irregular boundary surface. Several papers on scattering of elastic waves from boundary surfaces have appeared based on Rayleigh's method and other techniques, e.g. papers by Abubakar [13], Asano [14], Dunkin and Eringen [15], Gupta [16], Kuo and Nafe [17], Levy and Deresiewicz [18], Rice [19], Singh and Tomar [20, 21] and Tomar and Kaur [22].

Chattopadhyay et al. [23] investigated the problem of reflection and transmission of plane quasi Pwaves at a corrugated interface between distinct triclinic elastic half-spaces. They obtained the closed form expressions of reflection and transmission coefficients using Rayleigh's method of approximation. Kaur and Tomar [24] investigated the problem of reflection and transmission of shear wave incident upon a corrugated interface between two monoclinic elastic half-spaces with the help of Rayleigh's technique. Kennett [25] studied the problem of seismic wave scattering by obstacles on interfaces based on an integral equation formulation using the first-order perturbation theory. Paul and Campillo [26] investigated the effect of smallscale irregularities of a reflecting boundary on an elastic wave using a discretized form of boundary integral equations and a plane-wave decomposition of seismic wave fields. The scattering of plane harmonic $P, \mathrm{SV}$ or Rayleigh waves by two dimensions corrugated cavity completely embedded in an isotropic half-space or full-space was investigated by using a direct boundary integral method [27]. Singh and Singh [28] explained the problem of the effect of corrugation on an incident qSV-wave in pre-stressed elastic half-spaces with the help of Rayleigh's method of approximation. They found out the reflection and transmission coefficients of the regularly and irregularly reflected and transmitted waves. Singh and Tomar [29, 30] investigated the problem of qP-waves at a corrugated interface between two dissimilar monoclinic elastic half-spaces and obtained the reflection and transmission coefficients of the irregular waves using Rayleigh's technique.

In this paper, we have investigated the problem of reflection/transmission of qSV/qP-wave from a corrugated interface for an incident qSV-wave at a corrugated interface between two dissimilar monoclinic elastic half-spaces. Using Rayleigh's method of approximation, the expressions of the reflection and transmission coefficients of the irregular waves are obtained for the first order of approximation. These coefficients are derived and computed numerically for a special type of interface, $z=d \cos p y$. We have found that these coefficients are functions of elastic constants, angle of propagation, frequency and corrugation parameters.

\section{Basic equations}

The constitutive relations in a homogeneous anisotropic elastic material of monoclinic type with the $y z$-plane as the plane of symmetry are given by [11]

$$
\begin{aligned}
& \tau_{11}=c_{11} e_{11}+c_{12} e_{22}+c_{13} e_{33}+2 c_{14} e_{23}, \quad \tau_{22}=c_{12} e_{11}+c_{22} e_{22}+c_{23} e_{33}+2 c_{24} e_{23}, \\
& \tau_{23}=c_{14} e_{11}+c_{24} e_{22}+c_{34} e_{33}+2 c_{44} e_{23}, \quad \tau_{33}=c_{13} e_{11}+c_{23} e_{22}+c_{33} e_{33}+2 c_{34} e_{23}, \\
& \tau_{12}=2\left(c_{55} e_{13}+c_{56} e_{12}\right), \quad \tau_{13}=2\left(c_{56} e_{13}+c_{66} e_{12}\right),
\end{aligned}
$$

where $\boldsymbol{u}=\left(u_{1}, u_{2}, u_{3}\right)$ are components of displacement, $\tau_{i j}$ are stress tensors, $c_{i j}(i, j=1,2,3, \ldots, 6)$ are elastic constants and $e_{i j}$ is the strain tensor given by 


$$
e_{i j}=\frac{1}{2}\left(\frac{\partial u_{i}}{\partial x_{j}}+\frac{\partial u_{j}}{\partial x_{i}}\right)
$$

The equations of motion in such anisotropic materials without body forces are given by

$$
\frac{\partial \tau_{i j}}{\partial x_{j}}=\rho \frac{\partial^{2} u_{i}}{\partial t^{2}}, \quad(i, j=1,2,3)
$$

where $\rho$ is density of the medium.

Let us consider two dimensional wave propagation in the $y z$-plane so that

$$
u_{1}=0, \quad \frac{\partial}{\partial x_{1}} \equiv \frac{\partial}{\partial x} \equiv 0, \quad \frac{\partial}{\partial x_{2}} \equiv \frac{\partial}{\partial y} \quad \text { and } \quad \frac{\partial}{\partial x_{3}} \equiv \frac{\partial}{\partial z}
$$

The equations of motion in terms of displacements components may be written as

$$
\begin{aligned}
& c_{22} \frac{\partial^{2} u_{2}}{\partial y^{2}}+c_{44} \frac{\partial^{2} u_{2}}{\partial z^{2}}+c_{24} \frac{\partial^{2} u_{3}}{\partial y^{2}}+c_{34} \frac{\partial^{2} u_{3}}{\partial z^{2}}+2 c_{24} \frac{\partial^{2} u_{2}}{\partial y \partial z}+\left(c_{23}+c_{24}\right) \frac{\partial^{2} u_{3}}{\partial y \partial z}=\rho \frac{\partial^{2} u_{2}}{\partial t^{2}}, \\
& c_{24} \frac{\partial^{2} u_{2}}{\partial y^{2}}+c_{34} \frac{\partial^{2} u_{2}}{\partial z^{2}}+c_{44} \frac{\partial^{2} u_{3}}{\partial y^{2}}+c_{33} \frac{\partial^{2} u_{3}}{\partial z^{2}}+2 c_{34} \frac{\partial^{2} u_{3}}{\partial y \partial z}+\left(c_{23}+c_{24}\right) \frac{\partial^{2} u_{2}}{\partial y \partial z}=\rho \frac{\partial^{2} u_{3}}{\partial t^{2}},
\end{aligned}
$$

It may be noted that Eqs (2.3) and (2.4) are the equations of motion for the coupled qSV and qPwaves. The solution of these equations may be taken in the form

$$
\left\{u_{2}, u_{3}\right\}(y, z, t)=\left\{A d_{2}, A d_{3}\right\} \exp \left\{k\left(c t-p_{2} y-p_{3} z\right)\right\},
$$

where $c$ is the phase velocity, $k$ is the wavenumber, $\boldsymbol{p}=\left(0, p_{2}, p_{3}\right)$ is the unit propagation vector, $\boldsymbol{d}=$ $\left(0, d_{2}, d_{3}\right)$ is the unit displacement vector.

Using these expressions of $u_{2}$ and $u_{3}$ in Eqs (2.3) and (2.4), we have

$$
\left(X-\rho c^{2}\right) d_{2}+Y d_{3}=0, \quad Y d_{2}+\left(W-\rho c^{2}\right) d_{3}=0,
$$

where

$$
\begin{aligned}
& X=c_{22} p_{2}^{2}+c_{44} p_{3}^{2}+2 c_{24} p_{2} p_{3}, \quad Y=c_{24} p_{2}^{2}+c_{34} p_{3}^{2}+\left(c_{23}+c_{44}\right) p_{2} p_{3}, \\
& W=c_{44} p_{2}^{2}+c_{33} p_{3}^{2}+2 c_{34} p_{2} p_{3} .
\end{aligned}
$$

Using Eq.(2.6), we get

$$
2 \rho c_{2,1}^{2}=X+W \mp \sqrt{(X-W)^{2}+4 Y^{2}},
$$

where $(-v e)$ sign represents the phase velocity of $q S V$ - waves $\left(c_{2}\right)$ and $(+v e)$ sign represents that of $q P-$ waves $\left(c_{1}\right)$. 


\section{Problem formulation}

Consider the Cartesian coordinates with the $x$ and $y$-axis lying horizontal and the $z$-axis is vertical with positive direction pointing downward. Suppose two dissimilar homogeneous monoclinic half-spaces, $M=\{(y ; z): y \in R, z \in[\zeta, \infty)\}$ and $M^{\prime}=\{(y ; z): y \in R, z \in(-\infty, \zeta)\}$ are separated by $z=\zeta(y)$, which is a periodic function of $\mathrm{y}$ independent of $\mathrm{x}$ whose mean value is zero. We will denote all elastic constants, stress tensors and displacement components in medium $\mathrm{M}$ without prime and those of $\mathrm{M}^{\prime}$ with primes. The geometry of the problem is shown by Fig.1. The Fourier series expansion of $\zeta(y)$, is given as

$$
\zeta(y)=\sum_{n=1}^{\infty}\left(\zeta_{n} e^{\imath n p y}+\zeta_{-n} e^{-\imath n p y}\right)
$$

where $\zeta_{n}$ and $\zeta_{-n}$ are the coefficients of series expansion of order $n, p$ is the wavenumber and $1=\sqrt{-1}$. Introduce constants $d ; c_{n}$; and $s_{n}$ as

$$
\zeta_{ \pm l}=\frac{d}{2}, \quad \zeta_{ \pm n}=\frac{c_{n} \mp i s_{n}}{2}, \quad(n=2,3,4, \ldots \ldots)
$$

so that

$$
\zeta(y)=d \cos (p y)+\sum_{n=2}^{\infty}\left[c_{n} \cos (n p y)+s_{n} \sin (n p y)\right]
$$

If the interface is represented by only one cosine term, i.e., $\zeta(y)=d \cos (p y)$, then the wavelength of corrugation is $2 \pi / \rho$ and $\mathrm{d}$ is the amplitude of corrugation.

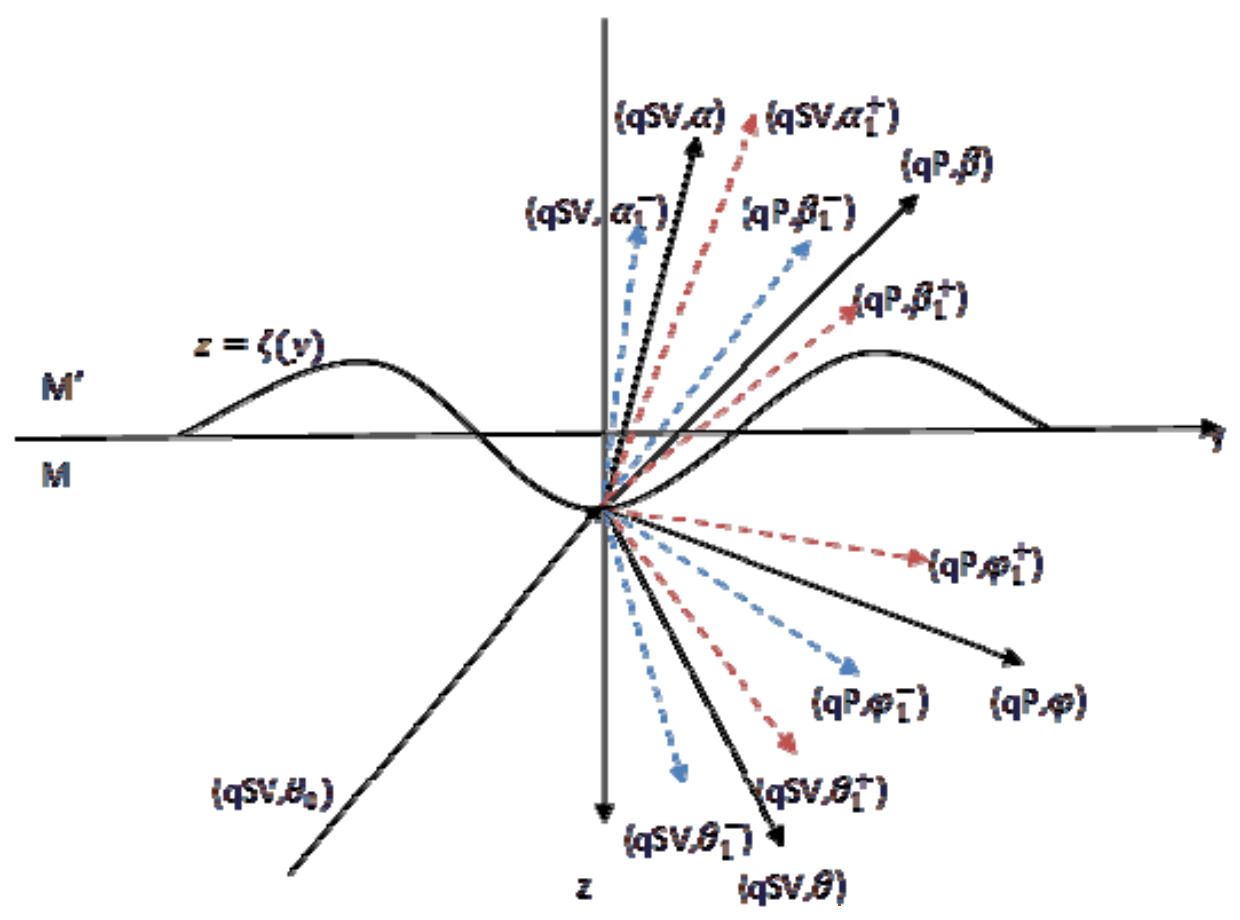

Fig.1. Geometry of the problem. 
We shall now discuss the reflection and transmission of elastic waves due to the incident plane qSVwave at the corrugated interface, $z=\zeta(y)$. Suppose a plane qSV-wave propagating in the half-space, $M$ with an angle $\theta_{0}$ and amplitude constant $A_{0}$ be incident at the corrugated interface. This incident wave gives rise to regularly and irregularly reflected and transmitted qSV and qP-waves [14].

The full structures of reflected and transmitted waves are given by:

(for the half-space, $M$ )

$$
\begin{aligned}
& u_{2}=A_{0} \exp \left(P_{0}^{\prime}\right)+A \exp (P)+B \exp (Q)+\sum_{n=1}^{\infty}\left\{A_{n}^{ \pm} \exp \left(P_{n}^{ \pm}\right)+B_{n}^{ \pm} \exp \left(Q_{n}^{ \pm}\right)\right\} \\
& u_{3}=D_{0} \exp \left(P_{0}^{\prime}\right)+D \exp (P)+E \exp (Q)+\sum_{n=1}^{\infty}\left\{D_{n}^{ \pm} \exp \left(P_{n}^{ \pm}\right)+E_{n}^{ \pm} \exp \left(Q_{n}^{ \pm}\right)\right\}
\end{aligned}
$$

where $(A, D)$ are amplitude constants of the regularly reflected qSV-wave at angle $\theta,\left(A_{n}^{ \pm}, D_{n}^{ \pm}\right)$are amplitude constants of the irregularly reflected qSV-waves at angles $\theta_{n}^{ \pm},(B, E)$ are amplitude constants of the regularly reflected $\mathrm{qP}$-wave at angle $\varphi,\left(B_{n}^{ \pm}, E_{n}^{ \pm}\right)$are amplitude constants of the irregularly reflected qP-wave at angles $\varphi_{n}^{ \pm}$and the expressions of $P_{0}^{\prime}, P, P_{n}^{ \pm}, \mathrm{Q}, Q_{n}^{ \pm}$are given by $P_{0}^{\prime}=\imath \omega\left(t-\frac{y \sin \theta_{0}-z \cos \theta_{0}}{c_{0}}\right)$, $P=1 \omega\left(t-\frac{y \sin \theta+z \cos \theta}{c_{1}}\right), P_{n}^{ \pm}=1 \omega\left(t-\frac{y \sin \theta_{n}^{ \pm}+z \cos \theta_{n}^{ \pm}}{c_{2}}\right), Q=i \omega\left(t-\frac{y \sin \varphi+z \cos \varphi}{c_{1}}\right)$ and $Q_{n}^{ \pm}=1 \omega\left(t-\frac{y \sin \varphi_{n}^{ \pm}+z \cos \varphi_{n}^{ \pm}}{c_{1}}\right)$.

These amplitude constants satisfy the following relations [11]

$$
A_{0}=F_{0} D_{0}, \quad A=F D, \quad B=F_{10} E, \quad A_{n}^{ \pm}=F_{n}^{ \pm} D_{n}^{ \pm}, \quad B_{n}^{ \pm}=F_{1 n}^{ \pm} E_{n}^{ \pm},
$$

where

$$
\begin{aligned}
F_{0} & =\frac{Y_{0}}{\rho c_{0}^{2}-X_{0}}, \quad F=\frac{Y_{10}}{\rho c_{0}^{2}-X_{10}}, \quad F_{10}=\frac{Y_{20}}{\rho c_{1}^{2}-X_{20}}, \quad F_{n}^{ \pm}=\frac{Y_{1 n}^{ \pm}}{\rho c_{2}^{2}-X_{1 n}^{ \pm}}, \quad F_{1 n}^{ \pm}=\frac{Y_{2 n}^{ \pm}}{\rho c_{1}^{2}-X_{2 n}^{ \pm}}, \\
2 \rho c_{0}^{2} & =X_{0}+W_{0}-\sqrt{\left(X_{0}-W_{0}\right)^{2}+4 Y_{0}^{2}}, \quad 2 \rho c_{2}^{2}=X_{10}+W_{10}-\sqrt{\left(X_{10}-W_{10}\right)^{2}+4 Y_{10}^{2}}, \\
2 \rho c_{1}^{2} & =X_{20}+W_{20}+\sqrt{\left(X_{20}-W_{20}\right)^{2}+4 Y_{20}^{2}} .
\end{aligned}
$$

(for the half-space, $M^{\prime}$ )

$$
\begin{aligned}
& u_{2}^{\prime}=G \exp (R)+H \exp (S)+\sum_{n=1}^{\infty}\left\{G_{n}^{ \pm} \exp \left(R_{n}^{ \pm}\right)+H_{n}^{ \pm} \exp \left(S_{n}^{ \pm}\right)\right\}, \\
& u_{3}^{\prime}=I \exp (R)+J \exp \left(P+\sum_{n=1}^{\infty}\left\{I_{n}^{ \pm} \exp \left(R_{n}^{ \pm}\right)+J_{n}^{ \pm} \exp \left(S_{n}^{ \pm}\right)\right\},\right.
\end{aligned}
$$


where $(G, I)$ are amplitude constants of the regularly transmitted qSV-wave at angle $\alpha,\left(G_{n}^{ \pm}, I_{n}^{ \pm}\right)$are amplitude constants of the irregularly transmitted qSV-waves at angles $\alpha_{n}^{ \pm},(H, J)$ are amplitude constants of the regularly transmitted qP-wave at angle $\beta,\left(H_{n}^{ \pm}, J_{n}^{ \pm}\right)$are amplitude constants of the irregularly transmitted qP-waves at angles $\beta_{n}^{ \pm}$and the expressions of $R, R_{n}^{ \pm}, S, S_{n}^{ \pm}$are given by $R=\imath \omega\left(t-\frac{y \sin \alpha-z \cos \alpha}{C_{2}^{\prime}}\right)$, $R_{n}^{ \pm}=1 \omega\left(t-\frac{y \sin \alpha_{n}^{ \pm}-z \cos \alpha_{n}^{ \pm}}{C_{2}^{\prime}}\right), S=\imath \omega\left(t-\frac{y \sin \beta-z \cos \beta}{C_{1}^{\prime}}\right)$ and $S_{n}^{ \pm}=1 \omega\left(t-\frac{y \sin \beta_{n}^{ \pm}-z \cos \beta_{n}^{ \pm}}{C_{1}^{\prime}}\right)$.

These amplitude constants also satisfy the following relations

where

$$
G=F_{20} I, \quad H=F_{30} J, \quad G_{n}^{ \pm}=F_{2 n}^{ \pm} I_{n}^{ \pm}, \quad H_{n}^{ \pm}=F_{3 n}^{ \pm} J_{n}^{ \pm},
$$

$$
\begin{aligned}
& F_{20}=\frac{Y_{30}}{\rho^{\prime} c_{2}^{\prime 2}-X_{30}}, \quad F_{30}=\frac{Y_{40}}{\rho^{\prime} c_{1}^{\prime 2}-X_{40}}, \quad F_{2 n}^{ \pm}=\frac{Y_{3 n}^{ \pm}}{\rho^{\prime} c_{2}^{\prime 2}-X_{3 n}^{ \pm}}, \quad F_{3 n}^{ \pm}=\frac{Y_{4 n}^{ \pm}}{\rho^{\prime} c_{1}^{\prime 2}-X_{4 n}^{ \pm}}, \\
& 2 \rho^{\prime} c_{2}^{\prime 2}=X_{30}+W_{30}-\sqrt{\left(X_{30}-W_{30}\right)^{2}+4 Y_{30}^{2}}, \quad 2 \rho^{\prime} c_{1}^{\prime 2}=X_{40}+W_{40}+\sqrt{\left(X_{40}-W_{40}\right)^{2}+4 Y_{40}^{2}} .
\end{aligned}
$$

The expressions of $X, Y$ and $W$ with corresponding suffixes are obtained from Eq.(2.7) by inserting $\left(p_{2}, p_{3}\right)$; for the incident qSV-wave: $\left(\sin \theta_{0},-\cos \theta_{0}\right)$, for the regularly reflected qSV -wave: $(\sin \theta, \cos \theta)$, for the irregularly reflected qSV-wave: $\left(\sin \theta_{n}^{ \pm}, \cos \theta_{n}^{ \pm}\right)$, for the regularly reflected qP-wave: $(\sin \varphi, \cos \varphi)$, for the irregularly reflected qP-wave: $\left(\sin \varphi_{n}^{ \pm}, \cos \varphi_{n}^{ \pm}\right)$, for the regular transmitted qSV-wave: $(\sin \alpha,-\cos \alpha)$; for the irregularly transmitted qSV -wave: $\left(\sin \alpha_{n}^{ \pm},-\cos \alpha_{n}^{ \pm}\right)$, for the regularly transmitted qP-wave: $(\sin \beta,-\cos \beta)$, for irregularly transmitted qP-wave: $\left(\sin \beta_{n}^{ \pm},-\cos \beta_{n}^{ \pm}\right)$.

The Snell law of this problem is given by [14]

$$
\frac{\sin \theta_{0}}{c_{0}\left(\theta_{0}\right)}=\frac{\sin \theta}{c_{2}(\theta)}=\frac{\sin \varphi}{C_{1}(\varphi)}=\frac{\sin \alpha}{C_{2}^{\prime}(\alpha)}=\frac{\sin \beta}{C_{l}^{\prime}(\beta)}=\frac{1}{c_{a}}
$$

where $c_{a}$ is apparent velocity.

'Moreover, spectrum theorem gives the relations between the angle of the regular wave and those of irregular waves [13]

$$
\sin \left\{\begin{array}{l}
\theta_{n}^{ \pm} \\
\varphi_{n}^{ \pm} \\
\alpha_{n}^{ \pm} \\
\beta_{n}^{ \pm}
\end{array}\right\}-\sin \left\{\begin{array}{l}
\theta \\
\varphi \\
\alpha \\
\beta
\end{array}\right\}= \pm \frac{n p}{\omega}\left\{\begin{array}{c}
c_{2} \\
c_{1} \\
c_{2} \\
c_{1}^{\prime}
\end{array}\right\}, n=0,1,2
$$

where $(+v e)$ signs of the right hand side correspond to $(+v e)$ signs of the left hand side, while $(-v e)$ signs of the right hand side correspond to $(-v e)$ signs of the left hand side of the equation. 


\section{Boundary condition}

The component of displacements and tractions (normal and shear) are continuous at the corrugated interface. Mathematically, these conditions at $z=\zeta(y)$ may be written as

$$
\begin{aligned}
& u_{2}=u_{2}^{\prime}, \quad u_{3}=u_{3}^{\prime}, \\
& \tau_{32}+\left(\tau_{33}-\tau_{22}\right) \zeta^{\prime}-\tau_{23} \zeta^{\prime 2}=\tau_{32}^{\prime}+\left(\tau_{33}^{\prime}-\tau_{22}^{\prime}\right) \zeta^{\prime}-\tau_{23}^{\prime} \zeta^{\prime 2}, \\
& \tau_{33}-2 \tau_{23} \zeta^{\prime}+\tau_{22} \zeta^{\prime 2}=\tau_{33}^{\prime}-2 \tau_{23}^{\prime} \zeta^{\prime}+\tau_{22}^{\prime} \zeta^{\prime 2},
\end{aligned}
$$

where $\zeta^{\prime}$ is the derivative of $\zeta$ with respect to $y$.

Inserting Eq.(2.1) into Eqs (4.2) and (4.3), we get

$$
\begin{aligned}
& \left\{\left(c_{23}-c_{22}\right) \zeta^{\prime}+c_{24}\left(1-\zeta^{\prime 2}\right)\right\} \frac{\partial u_{2}}{\partial y}+\left\{\left(c_{33}-c_{23}\right) \zeta^{\prime}+c_{34}\left(1-\zeta^{\prime 2}\right)\right\} \frac{\partial u_{3}}{\partial z}+ \\
& +\left\{\left(c_{34}-c_{24}\right) \zeta^{\prime}+c_{44}\left(1-\zeta^{\prime 2}\right)\right\}\left(\frac{\partial u_{2}}{\partial z}+\frac{\partial u_{3}}{\partial y}\right)=\left\{\left(c_{23}^{\prime}-c_{22}^{\prime}\right) \zeta^{\prime}+c_{24}^{\prime}\left(1-\zeta^{\prime 2}\right)\right\} \frac{\partial u_{2}^{\prime}}{\partial y}+ \\
& +\left\{\left(c_{33}^{\prime}-c_{23}^{\prime}\right) \zeta^{\prime}+c_{34}^{\prime}\left(1-\zeta^{\prime 2}\right)\right\} \frac{\partial u_{3}^{\prime}}{\partial z}+\left\{\left(c_{34}^{\prime}-c_{24}^{\prime}\right) \zeta^{\prime}+c_{44}^{\prime}\left(1-\zeta^{\prime 2}\right)\right\}\left(\frac{\partial u_{2}^{\prime}}{\partial z}+\frac{\partial u_{3}^{\prime}}{\partial y}\right) \\
& \left\{c_{23}+c_{22} \zeta^{\prime 2}-2 c_{24} \zeta^{\prime}\right\} \frac{\partial u_{2}}{\partial y}+\left\{c_{33}+c_{23} \zeta^{\prime 2}-2 c_{34} \zeta^{\prime}\right\} \frac{\partial u_{3}}{\partial z}+ \\
& +\left\{c_{34}+c_{24} \zeta^{\prime 2}-2 c_{44} \zeta^{\prime}\right\}\left(\frac{\partial u_{2}}{\partial z}+\frac{\partial u_{3}}{\partial y}\right)=\left\{c_{23}^{\prime}+c_{22}^{\prime} \zeta^{\prime 2}-2 c_{24}^{\prime} \zeta^{\prime}\right\} \frac{\partial u_{2}^{\prime}}{\partial y}+ \\
& +\left\{c_{33}^{\prime}+c_{23}^{\prime} \zeta^{\prime 2}-2 c_{34}^{\prime} \zeta^{\prime}\right\} \frac{\partial u_{3}^{\prime}}{\partial z}+\left\{c_{34}^{\prime}+c_{24}^{\prime} \zeta^{\prime 2}-2 c_{44}^{\prime} \zeta^{\prime}\right\}\left(\frac{\partial u_{2}^{\prime}}{\partial z}+\frac{\partial u_{3}^{\prime}}{\partial y}\right) .
\end{aligned}
$$

Using Eqs (3.3), (3.4), (3.6), (3.7), (3.9) and (3.10) in Eqs (4.1), (4.4) and (4.5), we get

$$
\begin{aligned}
& D_{0} e^{\imath \zeta K_{0}}+D e^{-\imath \zeta K}+E e^{-\imath \zeta L}+\sum_{n=1}^{\infty}\left\{D_{n}^{ \pm} e^{\mp \imath n p y-\imath \zeta K_{n}^{ \pm}}+E_{n}^{ \pm} e^{\mp \imath n p y-\imath \zeta L_{n}^{ \pm}}\right\}= \\
& =I e^{1 \zeta M}+J e^{1 \zeta N}+\sum_{n=1}^{\infty}\left\{I_{n}^{ \pm} e^{\mp 1 n p y+1 \zeta M_{n}^{ \pm}}+J_{n}^{ \pm} e^{\mp 1 n p y+1 \zeta N_{n}^{ \pm}}\right\}, \\
& A_{0} e^{1 \zeta K_{0}}+A e^{-1 \zeta K}+B e^{-1 \zeta L}+\sum_{n=1}^{\infty}\left\{A_{n}^{ \pm} e^{\mp \imath n p y-1 \zeta K_{n}^{ \pm}}+B_{n}^{ \pm} e^{\mp i n p y-1 \zeta L_{n}^{ \pm}}\right\}= \\
& =G e^{i \zeta M}+H e^{1 \zeta N}+\sum_{n=1}^{\infty}\left\{G_{n}^{ \pm} e^{\mp \imath n p y+1 \zeta M_{n}^{ \pm}}+H_{n}^{ \pm} e^{\mp \imath n p y+1 \zeta N_{n}^{ \pm}}\right\},
\end{aligned}
$$




$$
\begin{aligned}
& {\left[\left(c_{23}-c_{22}\right) \zeta^{\prime}+c_{24}\left(1-\zeta^{\prime 2}\right)\right]\left[P_{0} A_{0} e^{1 \zeta K_{0}}+P_{0} A e^{-1 \zeta K}+P_{0} B e^{-1 \zeta L}+\right.} \\
& \left.+\sum_{n=1}^{\infty}\left\{\left(P_{0} \pm n p\right) A_{n}^{ \pm} e^{\mp i n p y-1 \zeta K_{n}^{ \pm}}+\left(P_{0} \pm n p\right) B_{n}^{ \pm} e^{\mp i n p y-1 \zeta L_{n}^{ \pm}}\right\}\right]+\left[\left(c_{33}-c_{23}\right) \zeta^{\prime}+c_{34}\left(1-\zeta^{\prime 2}\right)\right]+ \\
& \times\left[-K_{0} D_{0} e^{1 \zeta K_{0}}+K D e^{-\imath \zeta K}+L E e^{-\imath \zeta L}+\sum_{n=1}^{\infty}\left\{K_{n}^{ \pm} D_{n}^{ \pm} e^{\mp \imath n p y-\imath \zeta K_{n}^{ \pm}}+L_{n}^{ \pm} E_{n}^{ \pm} e^{\mp n n p y-1 \zeta L_{n}^{ \pm}}\right\}\right]+ \\
& +\left[\left(c_{34}-c_{24}\right) \zeta^{\prime}+c_{44}\left(1-\zeta^{\prime 2}\right)\right]\left[-K_{0} A_{0} e^{1 \zeta K_{0}}+K A e^{-1 \zeta K}+L B e^{-\imath \zeta L}+P_{0} D_{0} e^{1 \zeta K_{0}}+P_{0} D e^{-1 \zeta K}+\right. \\
& +P_{0} E e^{-\imath \zeta L}+\sum_{n=1}^{\infty}\left\{K_{n}^{ \pm} A_{n}^{ \pm} e^{\mp \imath n p y-\imath \zeta K_{n}^{ \pm}}+L_{n}^{ \pm} B_{n}^{ \pm} e^{\mp \imath n p y-\imath \zeta L_{n}^{ \pm}}+\left(P_{0} \pm n p\right) D_{n}^{ \pm} e^{\mp \imath n p y-\imath \zeta K_{n}^{ \pm}}+\right. \\
& \left.\left.+\left(P_{0} \pm n p\right) E_{n}^{ \pm} e^{\mp 1 n p y-1 \zeta L_{n}^{ \pm}}\right\}\right]=\left[\left(c_{23}^{\prime}-c_{22}^{\prime}\right) \zeta^{\prime}+c_{24}^{\prime}\left(1-\zeta^{\prime 2}\right)\right]\left[P_{0} G e^{1 \zeta M}+P_{0} H e^{1 \zeta N}+\right. \\
& \left.+\sum_{n=1}^{\infty}\left\{\left(P_{0} \pm n p\right) G_{n}^{ \pm} e^{\mp 1 n p y+1 \zeta M_{n}^{ \pm}}+\left(P_{0} \pm n p\right) H_{n}^{ \pm} e^{\mp 1 n p y+1 \zeta N_{n}^{ \pm}}\right\}\right]-\left[\left(c_{33}^{\prime}-c_{23}^{\prime}\right) \zeta^{\prime}+c_{34}^{\prime}\left(1-\zeta^{\prime 2}\right)\right] \times \\
& \times\left[M I e^{1 \zeta M}+N J e^{1 \zeta N}+\sum_{n=1}^{\infty}\left\{M_{n}^{ \pm} I_{n}^{ \pm} e^{\mp 1 n p y+1 \zeta M_{n}^{ \pm}}+N_{n}^{ \pm} J_{n}^{ \pm} e^{\mp n n p y+1 \zeta N_{n}^{ \pm}}\right\}\right]-\left[\left(c^{\prime}{ }_{34}-c_{24}^{\prime}\right) \zeta^{\prime}+c_{44}^{\prime}\left(1-\zeta^{\prime 2}\right)\right] \\
& {\left[M G e^{1 \zeta M}+N H e^{1 \zeta N}-P_{0} I e^{1 \zeta M}-P_{0} J e^{\imath \zeta N}+\sum_{n=1}^{\infty}\left\{M_{n}^{ \pm} G_{n}^{ \pm} e^{\mp 1 n p y+1 \zeta M_{n}^{ \pm}}+\right.\right.} \\
& \left.\left.+N_{n}^{ \pm} H_{n}^{ \pm} e^{\mp \imath n p y+1 \zeta N_{n}^{ \pm}}-\left(P_{0} \pm n p\right) I_{n}^{ \pm} e^{\mp \imath n p y+1 \zeta M_{n}^{ \pm}}-\left(P_{0} \pm n p\right) J_{n}^{ \pm} e^{\mp i n p y+\imath \zeta N_{n}^{ \pm}}\right\}\right], \\
& {\left[c_{23}+c_{22} \zeta^{\prime 2}-2 c_{24} \zeta^{\prime}\right]\left[P_{0} A_{0} e^{\imath \zeta K_{0}}+P_{0} A e^{-\imath \zeta K}+P_{0} B e^{-\imath \zeta L}+\sum_{n=1}^{\infty}\left\{\left(P_{0} \pm n p\right) A_{n}^{ \pm} e^{\mp i n p y-\imath \zeta K_{n}^{ \pm}}+\right.\right.} \\
& \left.\left.+\left(P_{0} \pm n p\right) B_{n}^{ \pm} e^{\mp 1 n p y-1 \zeta L_{n}^{ \pm}}\right\}\right]+\left[c_{33}+c_{23} \zeta^{\prime 2}-2 c_{34} \zeta^{\prime}\right]\left[-K_{0} D_{0} e^{1 \zeta K_{0}}+K D e^{-1 \zeta K}+L E e^{-1 \zeta L}\right. \\
& \left.\sum_{n=1}^{\infty}\left\{K_{n}^{ \pm} D_{n}^{ \pm} e^{\mp i n p y-\imath \zeta K_{n}^{ \pm}}+L_{n}^{ \pm} E_{n}^{ \pm} e^{\mp i n p y-\imath \zeta L_{n}^{ \pm}}\right\}\right]+\left[c_{34}+c_{24} \zeta^{\prime 2}-2 c_{44} \zeta^{\prime}\right]\left[-K_{0} A_{0} e^{\imath \zeta K_{0}}+K A e^{-\imath \zeta K}+\right. \\
& +L B e^{-\imath \zeta L}+P_{0} D_{0} e^{\imath \zeta K_{0}}+P_{0} D e^{-\imath \zeta K}+P_{0} E e^{-\imath \zeta L}+\sum_{n=1}^{\infty}\left\{K_{n}^{ \pm} A_{n}^{ \pm} e^{\mp i n p y-\imath \zeta K_{n}^{ \pm}}+L_{n}^{ \pm} B_{n}^{ \pm} e^{\mp \imath n p y-\imath \zeta L_{n}^{ \pm}}+\right. \\
& \left.\left.+\left(P_{0} \pm n p\right) D_{n}^{ \pm} e^{\mp 1 n p y-1 \zeta K_{n}^{ \pm}}+\left(P_{0} \pm n p\right) E_{n}^{ \pm} e^{\mp n n p y-1 \zeta L_{n}^{ \pm}}\right\}\right]=\left[c_{23}^{\prime}+c_{22}^{\prime} \zeta^{\prime 2}-2 c_{24}^{\prime} \zeta^{\prime}\right] \times\left[P_{0} G e^{1 \zeta M}+P_{0} H e^{1 \zeta N}+\right. \\
& \left.\sum_{n=1}^{\infty}\left\{\left(P_{0} \pm n p\right) G_{n}^{ \pm} e^{\mp i n p y+1 \zeta M_{n}^{ \pm}}+\left(P_{0} \pm n p\right) H_{n}^{ \pm} e^{\mp 1 n p y-1 \zeta N_{n}^{ \pm}}\right\}\right]-\left[c_{33}^{\prime}+c_{23}^{\prime} \zeta^{\prime 2}-2 c_{34}^{\prime} \zeta^{\prime}\right] \\
& {\left[M I e^{1 \zeta M}+N J e^{1 \zeta N}+\sum_{n=1}^{\infty}\left\{M_{n}^{ \pm} I_{n}^{ \pm} e^{\mp 1 n p y+1 \zeta M_{n}^{ \pm}}+N_{n}^{ \pm} J_{n}^{ \pm} e^{\mp 1 n p y-1 \zeta N_{n}^{ \pm}}\right\}\right]-\left[c^{\prime}{ }_{34}+c^{\prime}{ }_{24} \zeta^{\prime 2}-2 c_{44} \zeta^{\prime}\right]} \\
& {\left[M G e^{1 \zeta M}+N H e^{1 \zeta N}-P_{0} I e^{1 \zeta M}-P_{0} J e^{1 \zeta N}+\sum_{n=1}^{\infty}\right\} M_{n}^{ \pm} G_{n}^{ \pm} e^{\mp 1 n p y+1 \zeta M_{n}^{ \pm}}+} \\
& \left.\left.+N_{n}^{ \pm} H_{n}^{ \pm} e^{\mp i n p y+1 \zeta N_{n}^{ \pm}}-\left(P_{0} \pm n p\right) I_{n}^{ \pm} e^{\mp i n p y+1 \zeta M_{n}^{ \pm}}-\left(P_{0} \pm n p\right) J_{n}^{ \pm} e^{\mp i n p y+1 \zeta N_{n}^{ \pm}}\right\}\right]
\end{aligned}
$$


where

$$
\begin{aligned}
& P_{0}=\frac{\omega \sin \theta_{0}}{c_{0}}, \quad K_{0}=\frac{\omega \cos \theta_{0}}{c_{0}}, \quad K=\frac{\omega \cos \theta}{c_{2}}, \quad K_{n}^{ \pm}=\frac{\omega \cos \theta_{n}^{ \pm}}{c_{2}}, \quad L=\frac{\omega \cos \varphi}{c_{1}}, \\
& L_{n}^{ \pm}=\frac{\omega \cos \varphi_{n}^{ \pm}}{c_{1}}, \quad M=\frac{\omega \cos \alpha}{c^{\prime}{ }_{2}}, \quad M_{n}^{ \pm}=\frac{\omega \cos \alpha_{n}^{ \pm}}{c^{\prime}{ }_{2}}, \quad N=\frac{\omega \cos \beta}{c^{\prime}{ }_{1}}, \quad N_{n}^{ \pm}=\frac{\omega \cos \beta_{n}^{ \pm}}{c^{\prime}{ }_{1}} .
\end{aligned}
$$

\section{Solution of first order approximation}

We assume that the amplitude of the corrugated interface is very small so that higher powers of $\zeta$ are neglected like

$$
e^{ \pm 1 \zeta K_{0}}=1 \pm 1 \zeta K_{0}-0\left(\zeta^{2}\right), \quad e^{ \pm 1 \zeta K}=1 \pm 1 \zeta K-0\left(\zeta^{2}\right)
$$

Using Eqs (3.5), (3.8) and (5.1) in Eqs (4.6)-(4.9) and collecting terms independent of $\zeta$ and y, we obtain a set of equations

$$
R S=T
$$

where

$$
\begin{aligned}
& R=\left[\begin{array}{cccc}
1 & 1 & -1 & -1 \\
F & F_{10}-F_{20} & -F_{30} \\
l_{1} & l_{2}-l_{3} & -l_{4} \\
m_{1} & m_{2}-m_{3} & -m_{4}
\end{array}\right], \quad S=\left[\begin{array}{c}
\frac{D}{D_{0}} \\
\frac{D}{D_{0}} \\
\frac{D}{D_{0}} \\
\frac{D}{D_{0}}
\end{array}\right], \quad \mathrm{T}=\left[\begin{array}{c}
-1 \\
-F_{0} \\
-l_{0} \\
-m_{0}
\end{array}\right], \\
& l_{0}=\left(F_{0} c_{24}+c_{44}\right) P_{0}-\left(F_{0} c_{44}+c_{34}\right) K_{0}, \quad l_{1}=\left(F_{24}+c_{44}\right) P_{0}+\left(F_{44}+c_{34}\right) K, \\
& l_{2}=\left(F_{10} c_{24}+c_{44}\right) P_{0}+\left(F_{10} c_{44}+c_{34}\right) L, \quad l_{3}=\left(F_{20}^{\prime} c_{24}+c_{44}^{\prime}\right) P_{0}-\left(F_{20} c_{44}^{\prime}+c_{34}^{\prime}\right) M, \\
& l_{4}=\left(F_{30} c_{24}^{\prime}+c_{44}^{\prime}\right) P_{0}-\left(F_{30} c_{44}^{\prime}+c_{34}^{\prime}\right) N, \quad m_{0}=\left(F_{0} c_{23}+c_{34}\right) P_{0}-\left(F_{0} c_{34}+c_{33}\right) K_{0}, \\
& m_{1}=\left(F_{0} c_{23}+c_{34}\right) P_{0}+\left(F_{0} c_{34}+c_{33}\right) K, \quad m_{2}=\left(F_{10} c_{23}+c_{34}\right) P_{0}+\left(F_{0} c_{34}+c_{33}\right) L, \\
& m_{3}=\left(F_{20} c_{23}^{\prime}+c_{34}^{\prime}\right) P_{0}-\left(F_{20} c_{34}^{\prime}+c_{33}^{\prime}\right) M, m_{4}=\left(F_{30} c_{23}^{\prime}+c_{34}^{\prime}\right) P_{0}-\left(F_{30} c_{34}^{\prime}+c_{33}^{\prime}\right) N .
\end{aligned}
$$

On solving Eq.(5.2), we get

$$
\frac{D}{D_{0}}=\frac{\Delta_{D}}{\Delta}, \quad \frac{E}{D_{0}}=\frac{\Delta_{E}}{\Delta}, \quad \frac{I}{D_{0}}=\frac{\Delta_{I}}{\Delta}, \quad \frac{J}{D_{0}}=\frac{\Delta_{J}}{\Delta},
$$


where

$$
\Delta=\left|\begin{array}{cccc}
1 & 1 & -1 & -1 \\
F & F_{10}-F_{20} & -F_{30} \\
l_{1} & l_{2} & -l_{3} & -l_{4} \\
m_{1} & m_{2}-m_{3} & -m_{4}
\end{array}\right|,
$$

and the values of $\Delta_{D}, \Delta_{E}, \Delta_{I}$ and $\Delta_{J}$ are obtained by replacing first, second, third and fourth column of $\Delta$ with column matrix, $T$ respectively. This equation gives the ratios of the amplitude constants corresponding to the vertical components of displacement.

The ratios of the amplitude constants corresponding to horizontal components of displacement is obtained with the help of Eqs (3.5), (3.8) and (5.3) as

$$
\frac{A}{A_{0}}=\frac{F}{F_{0}} \frac{\Delta_{D}}{\Delta}, \quad \frac{B}{A_{0}}=\frac{F_{10}}{F_{0}} \frac{\Delta_{E}}{\Delta}, \quad \frac{G}{A_{0}}=\frac{F_{20}}{F_{0}} \frac{\Delta_{I}}{\Delta}, \quad \frac{H}{A_{0}}=\frac{F_{30}}{F_{0}} \frac{\Delta_{J}}{\Delta} .
$$

Now, the amplitude of the incident qSV-wave is given by $\sqrt{A_{0}^{2}+D_{0}^{2}}=\sqrt{1+F_{0}^{2}} D_{0}$. Similarly, we find the amplitudes of reflected and transmitted qSV and qP-waves. Thus, the reflection and transmission coefficients of reflected and transmitted qSV and qP-waves for the incident qSV-wave are given by

$$
\begin{array}{ll}
r_{s v}=\sqrt{\frac{1+F^{2}}{1+F_{0}^{2}}} \frac{\Delta_{D}}{\Delta}, & r_{p}=\sqrt{\frac{1+F_{10}^{2}}{1+F_{0}^{2}}} \frac{\Delta_{E}}{\Delta}, \\
t_{s v}=\sqrt{\frac{1+F_{20}^{2}}{1+F_{0}^{2}}} \frac{\Delta_{I}}{\Delta}, \quad t_{p}=\sqrt{\frac{1+F_{30}^{2}}{1+F_{0}^{2}}} \frac{\Delta_{J}}{\Delta} .
\end{array}
$$

We come to know that these coefficients depend on elastic constants and the angle of incidence.

Next, comparing coefficients of $e^{\mp i n p y}$ on both sides of those equations, we get

$$
R^{\mp} S^{\mp}=T^{\mp}
$$

where

$$
\begin{aligned}
& R^{\mp}=\left[\begin{array}{cccc}
1 & 1 & -1 & -1 \\
F_{n}^{ \pm} & F_{1 n}^{ \pm}-F_{2 n}^{ \pm} & -F_{3 n}^{ \pm} \\
g_{5}^{\mp} & g_{6}^{\mp}-g_{7}^{\mp} & -g_{8}^{\mp} \\
h_{5}^{\mp} & h_{6}^{\mp}-h_{7}^{\mp} & -h_{8}^{\mp}
\end{array}\right], \quad S^{\mp}=\left[\begin{array}{c}
\frac{D_{n}^{ \pm}}{D_{0}} \\
\frac{E_{n}^{ \pm}}{D_{0}} \\
\frac{I_{n}^{ \pm}}{D_{0}} \\
\frac{J_{n}^{ \pm}}{D_{0}}
\end{array}\right], \quad T^{\mp}=\left[\begin{array}{c}
f_{1}^{\mp} \\
f_{2}^{\mp} \\
f_{3}^{\mp} \\
f_{4}^{\mp}
\end{array}\right], \\
& f_{1}^{\mp}=1 \zeta_{\mp n}\left[-K_{0}+K \frac{D}{D_{0}}+L \frac{E}{D_{0}}+M \frac{I}{D_{0}}+N \frac{J}{D_{0}}\right],
\end{aligned}
$$




$$
\begin{aligned}
& f_{2}^{\mp}=1 \zeta_{\mp n}\left[-F_{0} K_{0}+F K \frac{D}{D_{0}}+F_{10} L \frac{E}{D_{0}}+F_{20} M \frac{I}{D_{0}}+F_{30} N \frac{J}{D_{0}}\right] \\
& f_{3}^{\mp}=1\left[g_{0}^{\mp}+g_{1}^{\mp} \frac{D}{D_{0}}+g_{2}^{\mp} \frac{E}{D_{0}}-g_{3}^{\mp} \frac{I}{D_{0}}-g_{4}^{\mp} \frac{J}{D_{0}}\right], \\
& f_{4}^{\mp}=\mathrm{\imath}\left[h_{0}^{\mp}+h_{1}^{\mp} \frac{D}{D_{0}}+h_{2}^{\mp} \frac{E}{D_{0}}-h_{3}^{\mp} \frac{I}{D_{0}}-h_{4}^{\mp} \frac{J}{D_{0}}\right], \\
& g_{0}^{\mp}=\left[\left\{\mp\left(c_{23}-c_{22}\right) n p P_{0}+c_{24} P_{0} K_{0} \pm\left(c_{34}-c_{24}\right) n p K_{0}-c_{44} K_{0}^{2}\right\} F_{0} \pm\left(c_{33}-c_{23}\right) n p K_{0}+\right. \\
& \left.-c_{34} K_{0}^{2} \mp\left(c_{34}-c_{24}\right) n p P_{0}+c_{44} P_{0} K_{0}\right] \zeta_{\mp n}, \\
& g_{1}^{\mp}=\left[\left\{\mp\left(c_{23}-c_{22}\right) n p P_{0}-c_{24} P_{0} K \mp\left(c_{34}-c_{24}\right) n p K-c_{44} K^{2}\right\} F \mp\left(c_{33}-c_{23}\right) n p K+\right. \\
& \left.-c_{34} K^{2} \mp\left(c_{34}-c_{24}\right) n p P_{0}-c_{44} P_{0} K\right] \zeta_{\mp n} \text {, } \\
& g_{2}^{\mp}=\left[\left\{\mp\left(c_{23}-c_{22}\right) n p P_{0}-c_{24} P_{0} L \mp\left(c_{34}-c_{24}\right) n p L-c_{44} L^{2}\right\} F_{10} \mp\left(c_{33}-c_{23}\right) n p L+\right. \\
& \left.-c_{34} L^{2} \mp\left(c_{34}-c_{24}\right) n p P_{0}-c_{44} P_{0} L\right] \zeta_{\mp n}, \\
& g_{3}^{\mp}=\left[\left\{\mp\left(c_{23}^{\prime}-c_{22}^{\prime}\right) n p P_{0}+c_{24}^{\prime} P_{0} M \pm\left(c_{34}^{\prime}-c_{24}^{\prime}\right) n p M-c_{44}^{\prime} M^{2}\right\} F_{20} \pm\left(c_{33}^{\prime}-c_{23}^{\prime}\right) n p M+\right. \\
& \left.-c^{\prime}{ }_{34} M^{2} \mp\left(c^{\prime}{ }_{34}-c^{\prime}{ }_{24}\right) n p P_{0}+c^{\prime}{ }_{44} P_{0} M\right] \zeta_{\mp n} \text {, } \\
& g_{4}^{\mp}=\left[\left\{\mp\left(c_{23}^{\prime}-c_{22}^{\prime}\right) n p P_{0}+c_{24}^{\prime} P_{0} N \pm\left(c_{34}^{\prime}-c_{24}^{\prime}\right) n p N-c_{44}^{\prime} N^{2}\right\} F_{30} \pm\left(c_{33}^{\prime}-c_{23}^{\prime}\right) n p N+\right. \\
& \left.-c^{\prime}{ }_{34} N^{2} \mp\left(c^{\prime}{ }_{34}-c^{\prime}{ }_{24}\right) n p P_{0}+c_{44}^{\prime} P_{0} N\right] \zeta_{\mp n} \text {, } \\
& g_{5}^{\mp}=-\left[\left\{c_{44} K_{n}^{ \pm}+c_{24}\left(P_{0} \pm n p\right)\right\} F_{n}^{ \pm}+c_{34} K_{n}^{ \pm}+c_{44}\left(P_{0} \pm n p\right)\right] \\
& g_{6}^{\mp}=-\left[\left\{c_{44} L_{n}^{ \pm}+c_{24}\left(P_{0} \pm n p\right)\right\} F_{1 n}^{ \pm}+c_{34} L_{n}^{ \pm}+c_{44}\left(P_{0} \pm n p\right)\right], \\
& g_{7}^{\mp}=\left\{c_{44}^{\prime} M_{n}^{ \pm}-c_{24}^{\prime}\left(P_{0} \pm n p\right)\right\} F_{2 n}^{ \pm}+c_{34}^{\prime} M_{n}^{ \pm}-c_{44}^{\prime}\left(P_{0} \pm n p\right), \\
& g_{8}^{\mp}=\left\{c_{44}^{\prime} N_{n}^{ \pm}-c_{24}^{\prime}\left(P_{0} \pm n p\right)\right\} F_{3 n}^{ \pm}+c^{\prime}{ }_{34} N_{n}^{ \pm}-c_{44}^{\prime}\left(P_{0} \pm n p\right),
\end{aligned}
$$




$$
\begin{aligned}
& h_{0}^{\mp}=\left[\left\{c_{23} P_{0} K_{0} \pm 2 c_{24} n p P_{0}-c_{34} K_{0}^{2}-2 c_{44} n p K_{0}\right\} F_{0}+\right. \\
& \left.-c_{33} K_{0}^{2} \mp 2 c_{34} n p K_{0}-c_{34} P_{0} K_{0} \pm 2 c_{44} n p P_{0}\right] \zeta_{\mp n} \text {, } \\
& h_{1}^{\mp}=\left[\left\{-c_{23} P_{0} K \pm 2 c_{24} n p P_{0}-c_{34} K^{2} \pm 2 c_{44} n p K\right\} F-c_{33} K^{2} \pm 2 c_{34} n p K-c_{34} P_{0} K \pm 2 c_{44} n p P_{0}\right] \zeta_{\mp n}, \\
& h_{2}^{\mp}=\left[\left\{-c_{23} L \pm 2 c_{24} n p P_{0}-c_{34} L^{2} \pm 2 c_{44} n p L\right\} F_{10}-c_{33} L^{2} \pm 2 c_{34} n p L-c_{34} P_{0} L \pm 2 c_{44} n p P_{0}\right] \zeta_{\mp n}, \\
& h_{3}^{\mp}=\left[\left\{c^{\prime}{ }_{23} P_{0} M \pm 2 c^{\prime}{ }_{24} n p P_{0}-c^{\prime}{ }_{34} M^{2} \mp 2 c^{\prime}{ }_{44} n p M\right\} F_{20}+\right. \\
& \left.-c^{\prime}{ }_{33} M^{2} \mp 2 c^{\prime}{ }_{34} n p M+c^{\prime}{ }_{34} P_{0} M \pm 2 c^{\prime}{ }_{44} n p P_{0}\right] \zeta_{\mp n} \text {, } \\
& h_{4}^{\mp}=\left[\left\{c^{\prime}{ }_{23} P_{0} N \pm 2 c^{\prime}{ }_{24} n p P_{0}-c^{\prime}{ }_{34} N^{2} \mp 2 c^{\prime}{ }_{44} n p N\right\} F_{30}+\right. \\
& \left.-c^{\prime}{ }_{33} N^{2} \mp 2 c^{\prime}{ }_{34} n p N+c^{\prime}{ }_{34} P_{0} N \pm 2 c^{\prime}{ }_{44} n p P_{0}\right] \zeta_{\mp n} \text {, } \\
& h_{5}^{\mp}=-\left[\left\{c_{23}\left(P_{0} \pm n p\right)+c_{34} K_{n}^{ \pm}\right\} F_{n}^{ \pm}+c_{33} K_{n}^{ \pm}+c_{34}\left(P_{0} \pm n p\right)\right], \\
& h_{6}^{\mp}=-\left[\left\{c_{23}\left(P_{0} \pm n p\right)+c_{34} L_{n}^{ \pm}\right\} F_{l n}^{ \pm}+c_{33} L_{n}^{ \pm}+c_{34}\left(P_{0} \pm n p\right)\right], \\
& h_{7}^{\mp}=\left\{-c^{\prime}{ }_{23}\left(P_{0} \pm n p\right)+c^{\prime}{ }_{34} M_{n}^{ \pm}\right\} F_{2 n}^{ \pm}+c^{\prime}{ }_{33} M_{n}^{ \pm}-c^{\prime}{ }_{34}\left(P_{0} \pm n p\right), \\
& h_{8}^{\mp}=\left\{-c^{\prime}{ }_{23}\left(P_{0} \pm n p\right)+c^{\prime}{ }_{34} N_{n}^{ \pm}\right\} F_{3 n}^{ \pm}+c^{\prime}{ }_{33} N_{n}^{ \pm}-c^{\prime}{ }_{34}\left(P_{0} \pm n p\right) \text {. }
\end{aligned}
$$

Solving Eq.(5.7), we get

where

$$
\frac{D_{n}^{ \pm}}{D_{0}}=\frac{\Delta_{D_{n}^{ \pm}}}{\Delta^{ \pm}}, \quad \frac{E_{n}^{ \pm}}{D_{0}}=\frac{\Delta_{E_{n}^{ \pm}}}{\Delta^{ \pm}}, \quad \frac{I_{n}^{ \pm}}{D_{0}}=\frac{\Delta_{I_{n}^{ \pm}}}{\Delta^{ \pm}}, \quad \frac{J_{n}^{ \pm}}{D_{0}}=\frac{\Delta_{J_{n}^{ \pm}}}{\Delta^{ \pm}}
$$

$$
\Delta^{ \pm}=\left[\begin{array}{cccc}
1 & 1 & -1 & -1 \\
F_{n}^{ \pm} & F_{1 n}^{ \pm}-F_{2 n}^{ \pm} & -F_{3 n}^{ \pm} \\
g_{5}^{\mp} & g_{6}^{\mp}-g_{7}^{\mp} & -g_{8}^{\mp} \\
h_{5}^{\mp} & h_{6}^{\mp}-h_{7}^{\mp} & -h_{8}^{\mp}
\end{array}\right],
$$

and the values of $\Delta_{D_{n}^{ \pm}}, \Delta_{E_{n}^{ \pm}}, \Delta_{I_{n}^{ \pm}}$and $\Delta_{J_{n}^{ \pm}}$are obtained by replacing first, second, third and fourth column of $\Delta^{ \pm}$with column matrix, $T^{\mp}$ respectively. This equation gives the ratios of the amplitude constants of irregular waves corresponding to the vertical components of the displacement.

The ratios of the amplitude constants of irregular waves corresponding to horizontal components of displacement are obtained with the help of Eqs (3.5), (3.8) and (5.8) as 


$$
\frac{A_{n}^{ \pm}}{A_{0}}=\frac{F_{n}^{ \pm}}{F_{0}} \frac{\Delta_{D_{n}^{ \pm}}}{\Delta^{ \pm}}, \quad \frac{B_{n}^{ \pm}}{A_{0}}=\frac{F_{1 n}^{ \pm}}{F_{0}} \frac{\Delta_{E_{n}^{ \pm}}}{\Delta^{ \pm}}, \quad \frac{G_{n}^{ \pm}}{A_{0}}=\frac{F_{2 n}^{ \pm}}{F_{0}} \frac{\Delta_{I_{n}^{ \pm}}}{\Delta^{ \pm}}, \quad \frac{H_{n}^{ \pm}}{A_{0}}=\frac{F_{3 n}^{ \pm}}{F_{0}} \frac{\Delta_{J_{n}^{ \pm}}}{\Delta^{ \pm}} .
$$

The reflection and transmission coefficients of the first order of approximation for irregularly reflected and transmitted $\mathrm{qSV}$ and $\mathrm{qP}$-waves are

$$
\begin{array}{ll}
r_{s v^{ \pm}}^{n}=\sqrt{\frac{1+F_{n}^{ \pm^{2}}}{1+F_{0}^{2}}} \frac{\Delta_{D_{n}^{ \pm}}}{\Delta^{ \pm}}, \quad r_{p^{ \pm}}^{n}=\sqrt{\frac{1+F_{1 n}^{ \pm^{2}}}{1+F_{0}^{2}}} \frac{\Delta_{E_{n}^{ \pm}}}{\Delta^{ \pm}}, \\
t_{s v^{ \pm}}^{n}=\sqrt{\frac{1+F_{2 n}^{ \pm^{2}}}{1+F_{0}^{2}}} \frac{\Delta_{I_{n}^{ \pm}}}{\Delta^{ \pm}}, \quad t_{p^{ \pm}}^{n}=\sqrt{\frac{1+F_{3 n}^{ \pm^{2}}}{1+F_{0}^{2}}} \frac{\Delta_{J_{n}^{ \pm}}}{\Delta^{ \pm}} .
\end{array}
$$

We come to know from Eqs (5.10) that the coefficients corresponding to the irregularly reflected and transmitted qSV and qP-waves are functions of the elastic constants, angle of incidence, corrugation and frequency parameters.

\section{Special case: An interface of $z=d \cos p y$}

When the interface is represented by only one cosine term, $z=d \cos p y$, with $\mathrm{d}$ as the amplitude of corrugation. In this case

$$
\zeta_{-n}=\zeta_{n}=\left\{\begin{array}{lll}
0 & \text { if } & n \neq 1 \\
\frac{d}{2} & \text { if } & n=1
\end{array}\right.
$$

Thus, using these values, the reflection and transmission coefficients for the first order approximation of the corrugation are given by

$$
\begin{array}{ll}
r_{s v^{ \pm}}^{1}=\sqrt{\frac{1+F_{1}^{ \pm^{2}}}{1+F_{0}^{2}}} \frac{\Delta_{D_{I}^{ \pm}}}{\Delta^{ \pm}}, & r_{p^{ \pm}}^{1}=\sqrt{\frac{1+F_{11}^{ \pm^{2}}}{1+F_{0}^{2}}} \frac{\Delta_{E_{I}^{ \pm}}}{\Delta^{ \pm}}, \\
t_{s v^{ \pm}}^{1}=\sqrt{\frac{1+F_{21}^{ \pm^{2}}}{1+F_{0}^{2}}} \frac{\Delta_{I_{I}^{ \pm}}}{\Delta^{ \pm}}, & t_{p^{ \pm}}^{1}=\sqrt{\frac{1+F_{31}^{ \pm^{2}}}{1+F_{0}^{2}}} \frac{\Delta_{J_{I}^{ \pm}}}{\Delta^{ \pm}}
\end{array}
$$

where values of $F_{I}^{ \pm}, F_{1 I}^{ \pm}, F_{2 l}^{ \pm}, F_{31}^{ \pm}, \Delta^{ \pm}, \Delta_{D_{I}^{ \pm}}, \Delta_{E_{I}^{ \pm}}, \Delta_{I_{I}^{ \pm}}$and $\Delta_{J_{I}^{ \pm}}$are obtained from Eq.(5.10) by using Eq.(6.1). We will compute these coefficients for a particular model.

\section{Particular case}

(a) When the two monoclinic half-spaces, $M$ and $M$ reduce to transversely isotropic half-spaces with the axis of symmetry coinciding with the $x$-axis, we have 


$$
\begin{gathered}
c_{12}=c_{13}, \quad c_{22}=c_{33}, \quad c_{55}=c_{66}, \quad c_{23}=c_{22}-2 c_{44}, \quad c_{14}=c_{24}=c_{34}=c_{56}=0, \\
c_{12}^{\prime}=c_{13}^{\prime}, \quad c_{22}^{\prime}=c_{33}^{\prime}, \quad c_{55}^{\prime}=c_{66}^{\prime}, \quad c_{23}^{\prime}=c_{22}^{\prime}-2 c^{\prime}{ }_{44}, \quad c_{14}^{\prime}=c^{\prime}{ }_{24}=c_{34}^{\prime}=c^{\prime}{ }_{56}=0 .
\end{gathered}
$$

Using these values in Eqs (5.5), (5.6) and (5.10), we may obtain the reflection and transmission coefficients corresponding to the regular and irregular waves.

(b) If the corrugation of the interface is neglected, i.e., $d=0$, the problem reduces to the reflection and transmission of elastic waves at a plane interface between two monoclinic elastic half-spaces. The reflection and transmission coefficients of the reflected and transmitted qSV and qP-waves are given by Eqs (5.5) and (5.6). These results exactly match those of Singh and Khurana [11].

(c) If the half-space, $M^{\prime}$ is absent, then the problem reduces to the reflection of qSV and qP-waves for the incident qSV-wave. The reflection coefficients are given by Eq.(5.5) with the following modified values

$$
\Delta=l_{1} m_{2}-l_{2} m_{1}, \quad \Delta_{D}=l_{2} m_{0}-l_{0} m_{2}, \quad \Delta_{E}=l_{0} m_{1}-l_{1} m_{0} .
$$

These results exactly match those of Singh and Khurana [6].

\section{Numerical results and discussion}

We will compute the angles of reflected and transmitted waves through Snell's law given by Eq.(3.9) in which the apparent velocity $c_{a}$ is related with the dimensionless velocity by $\bar{c}=\frac{c_{a}}{\lambda}$. Let us find out the angles of reflected qSV and qP-waves in the half-space, $M$. Equation (2.8) may be written as

$$
\bar{c}^{4}-(\bar{W}+\bar{X}) \bar{c}^{2}+\left(\overline{W X}-\bar{Y}^{2}\right)=0
$$

where

$$
\bar{X}=\frac{X}{p_{2}^{2} c_{44}}, \quad \bar{Y}=\frac{Y}{p_{2}^{2} c_{44}}, \quad \bar{W}=\frac{W}{p_{2}^{2} c_{44}}, \quad \lambda=\sqrt{\frac{c_{44}}{\rho}}, \quad \bar{c}_{i j}=\frac{c_{i j}}{c_{44}} .
$$

There are two roots of $\bar{c}^{2}$ corresponding to $\mathrm{qSV}$ and $\mathrm{qP}$-waves for a given $p=\frac{p_{3}}{p_{2}}$ and for a given value of $\bar{c}^{2}$, there are two roots of p corresponding to the angles of reflected qSV and qP-waves [29]. Substituting the values of $\bar{X}, \bar{Y}$, and $\bar{W}$ into Eq.(8.1), we get

where

$$
d_{0} p^{4}+d_{1} p^{3}+d_{2} p^{2}+d_{3} p+d_{4}=0
$$

$$
\begin{aligned}
& d_{0}=\bar{c}_{33}-\bar{c}_{34}^{2}, \quad d_{1}=2\left(\bar{c}_{24} \bar{c}_{33}-\bar{c}_{23} \bar{c}_{34}\right), \quad d_{2}=1+\bar{c}_{22} \bar{c}_{33}+2 \bar{c}_{24} \bar{c}_{34}-\left(1+\bar{c}_{23}\right)^{2}-\left(1+\bar{c}_{33}\right) \bar{c}^{2}, \\
& d_{3}=2\left\{\bar{c}_{22} \bar{c}_{34}-\bar{c}_{23} \bar{c}_{24}-\left(\bar{c}_{24}+\bar{c}_{34}\right) \bar{c}^{2}\right\}, \quad d_{4}=\bar{c}^{4}-\left(1+\bar{c}_{22}\right) \bar{c}^{2}+\bar{c}_{22}-\bar{c}_{24}^{2} .
\end{aligned}
$$

We transform this equation with $q=\frac{1}{p}=\frac{p_{2}}{p_{3}}$ so that 


$$
d_{4} q^{4}+d_{3} q^{3}+d_{2} q^{2}+d_{1} q+d_{0}=0
$$

This equation has two positive roots, i.e., a smaller positive root $\left(q_{1}\right)$ and a larger positive root $\left(q_{2}\right)$ which represent the directions of reflected $\mathrm{qSV}$ and $\mathrm{qP}$-waves respectively. Thus, $\theta=\tan ^{-1}\left(q_{1}\right)$ and $\varphi=\tan ^{-1}\left(q_{2}\right)$.

Similarly, in the half-space $M^{\prime}$, the angles of transmitted qSV and qP-waves are obtained as

$$
\alpha=\tan ^{-1}\left(q^{\prime}{ }_{1}\right) \quad \text { and } \quad \beta=\tan ^{-1}\left(q^{\prime}{ }_{2}\right) .
$$

For the numerical computation, the following relevant values of elastic constants are taken [30]: (for half-space, $M$-Lithium tantalate)

$$
\begin{aligned}
& c_{24}=0.11 \times 10^{11} \mathrm{~N} / \mathrm{m}^{2}, \quad c_{23}=0.81 \times 10^{11} \mathrm{~N} / \mathrm{m}^{2}, \quad c_{34}=0, \quad c_{44}=0.94 \times 10^{11} \mathrm{~N} / \mathrm{m}^{2}, \\
& c_{33}=2.75 \times 10^{11} \mathrm{~N} / \mathrm{m}^{2}, \quad c_{22}=2.33 \times 10^{11} \mathrm{~N} / \mathrm{m}^{2}, \quad \rho=7400 \mathrm{Kg} / \mathrm{m}^{2} .
\end{aligned}
$$

(for half-space, $M^{\prime}$-Lithium neobate)

$$
\begin{aligned}
& c_{24}^{\prime}=-0.09 \times 10^{11} \mathrm{~N} / \mathrm{m}^{2}, \quad c_{23}^{\prime}=0.75 \times 10^{11} \mathrm{~N} / \mathrm{m}^{2}, \quad c_{34}^{\prime}=0, \quad c_{44}^{\prime}=1.06 \times 10^{11} \mathrm{~N} / \mathrm{m}^{2}, \\
& c_{33}^{\prime}=2.45 \times 10^{11} \mathrm{~N} / \mathrm{m}^{2}, \quad c_{22}^{\prime}=2.03 \times 10^{11} \mathrm{~N} / \mathrm{m}^{2}, \quad \rho^{\prime}=4700 \mathrm{Kg} / \mathrm{m}^{2}
\end{aligned}
$$

the corrugation parameter, $(c o r=p d)=0.0001$ and the frequency parameter, $f r=\frac{\omega}{p c_{0}}=100$ are taken unless otherwise mentioned. Figure 2 represents the variation of angles of reflected and transmitted qSV and $\mathrm{qP}$-waves with angle of incidence. We have seen here that the angle of incidence is not equal to the angle of reflection. Figures 3-11 represent the variation of reflection and transmission coefficients with the angle of incidence for different values of corrugation and frequency parameters, Figs 12-14 represent the variation of reflection and transmission coefficients with the corrugation parameter and Figs 15-17 represent the variation of reflection and transmission coefficients with the frequency parameter at $\theta=20^{\circ}$.

In Fig.2, all angles, $(\theta, \varphi, \alpha, \beta)$, of the regularly reflected and transmitted waves increase with the increase of the angle of incidence $\left(\theta_{0}\right)$. It is observed that the angles of reflection and transmission for qSVwaves are less than that of the qP-waves. Curve I in Fig.3 shows that $r_{s v}$ starts from a certain value which decreases to zero at $\theta_{0}=15^{0}$ and then increases up to $\theta_{0}=51^{0}$ with the increase of $\theta_{0}$. Thereafter, it decreases, touching zero value at $\theta_{0}=87^{\circ}$. In the same figure, Curve II shows that $r_{p}$ is parabolic in the region $0 \leq \theta_{0} \leq 35^{\circ}$ and then it increases with the increase of $\theta_{0}$. Curve III shows the decreasing nature of $t_{s v}$ up to $\theta_{0}=81^{\circ}$ and then it increases with the increase of $\theta_{0}$, while Curve IV shows that $t_{p}$ increases with the increase of $\theta_{0}$. 


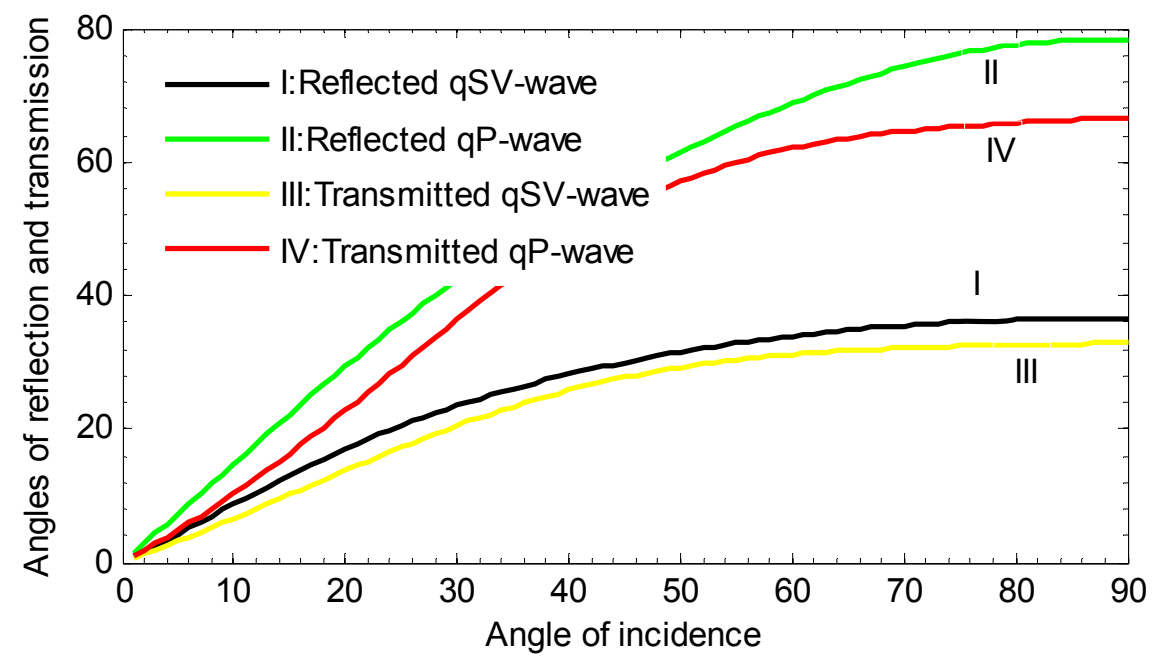

Fig.2. Variation of the angle of reflection and transmission with the angle of incidence.

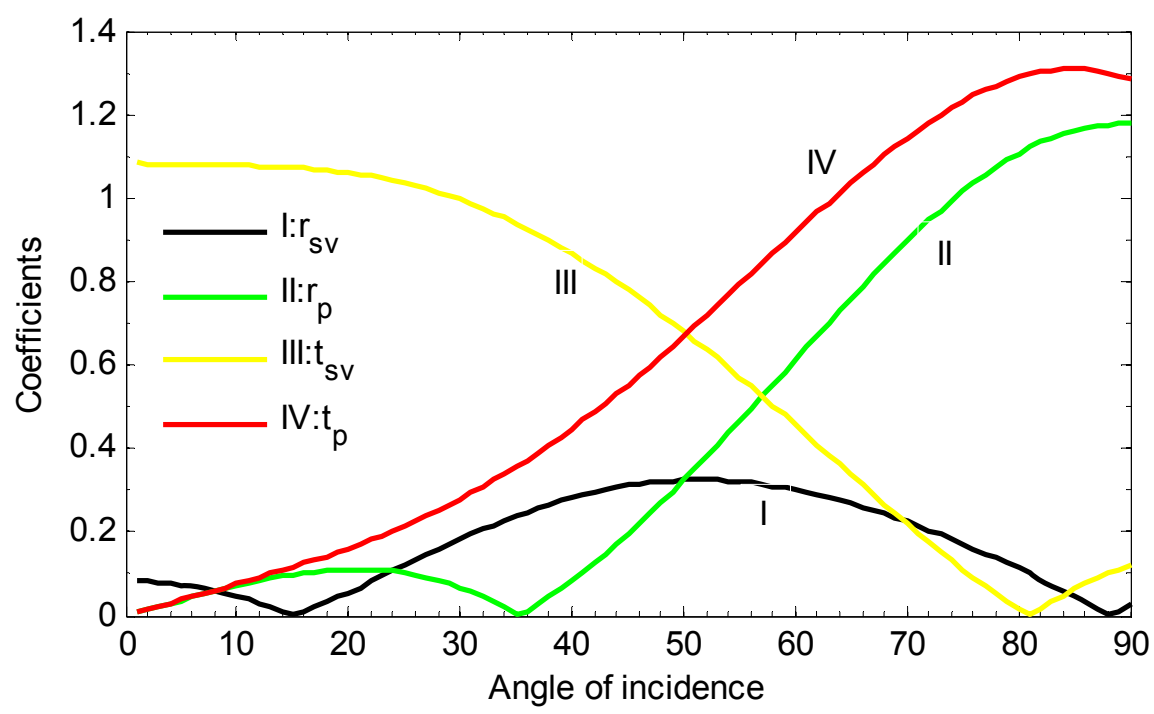

Fig.3. Variation of reflection and transmission coefficients with angle of incidence $\left(\theta_{0}\right)$.

\subsection{Effect of corrugation and frequency parameters}

We are interested to see the effect of corrugation and frequency parameters on the reflection and transmission coefficients. In Fig.4, the reflection coefficient, $r_{s v^{+}}^{1}$ corresponding to the irregularly reflected qSV-wave starts from a certain value and decreases to zero at $\theta_{0}=14^{0}$ creating a parabolic region in 1 $4^{0} \leq \theta_{0} \leq 81^{0}$, which then increases with the increase of $\theta_{0}$. It is observed that $r_{s v^{+}}^{1}$ increases with the increase of corrugation (cor) and frequency (fr) parameters. In Fig.5, $r_{p^{+}}^{1}$ creates two parabolic regions in $\$ 0$ $\leq \theta_{0} \leq 34^{0}$ and $34^{0} \leq \theta_{0} \leq 90^{\circ}$ with the increase of $\theta_{0}$. The values of this coefficient also increase with the 
increase of cor and fr. We have observed a similar nature of $r_{s v^{-}}^{l}$ and $r_{p^{-}}^{l}$ with $r_{s v^{+}}^{l}$ and $r_{p^{+}}^{l}$ respectively in Figs 6 and 7. The coefficient, $t_{s v^{+}}^{1}$ in Fig.8 decreases initially creating a parabolic region in $37^{0} \leq \theta_{0} \leq 67^{0}$ and then increases with the increase of $\theta_{0}$. It is observed that the values of $t_{s v^{+}}^{1}$ increase with the increase of cor and fr. We come to know that $t_{p^{+}}^{1}$ in Fig.9 creates a parabolic region in $2^{0} \leq \theta_{0} \leq 64^{0}$ and then increases with the increase of $\theta_{0}$. The value of this coefficient increases with the increase of cor and fr. Similar natures of $t_{s v^{-}}^{1}$ and $t_{P^{-}}^{1}$ with $t_{s v^{+}}^{1}$ and $t_{p^{+}}^{1}$ respectively are observed in Figs 10 and 11.

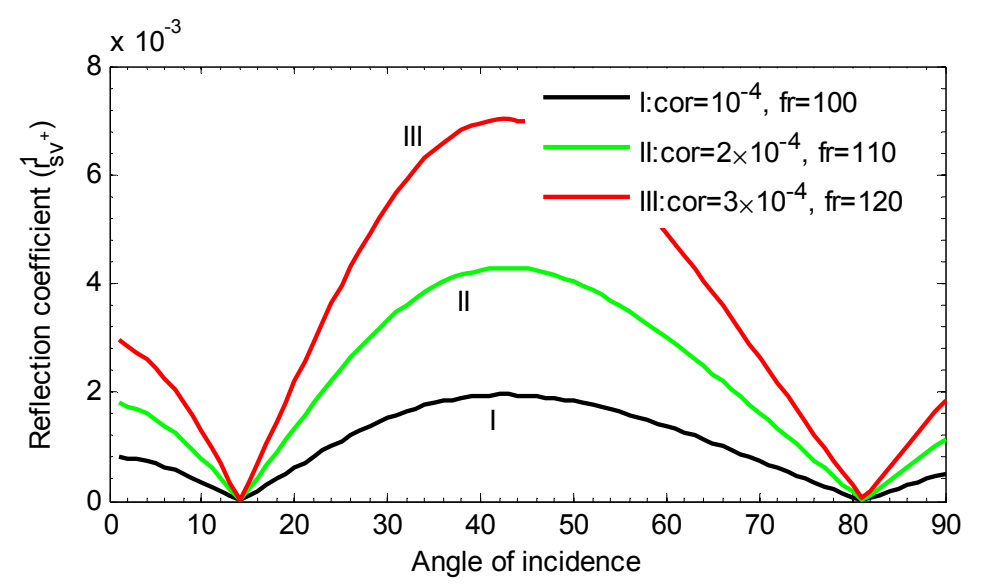

Fig.4. Variation of $r_{s v^{+}}^{1}$ with $\theta_{0}$ for different values of cor and $f r$.

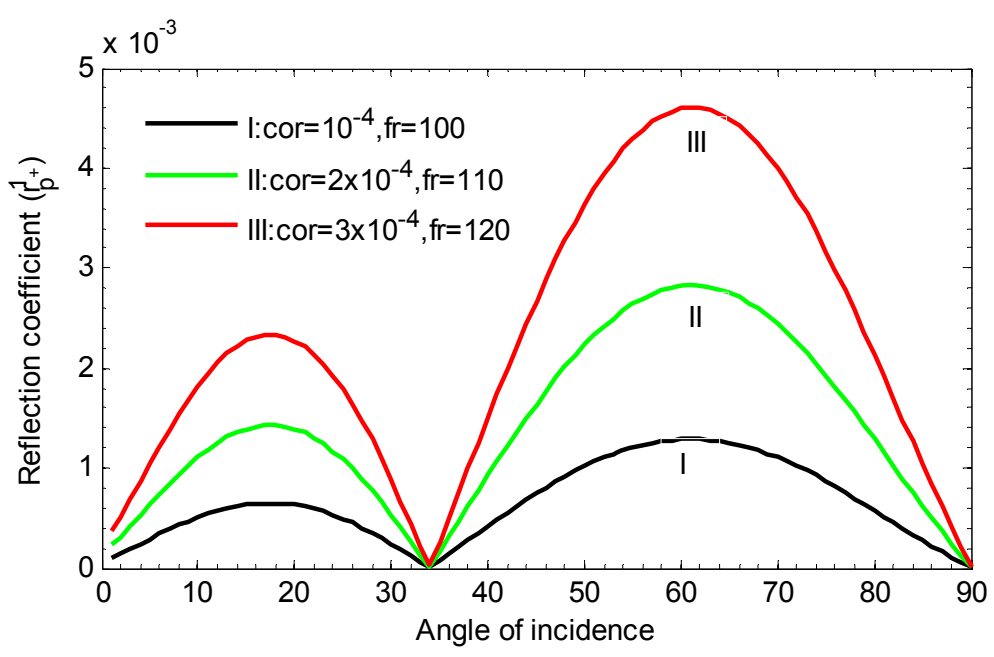

Fig.5. Variation of $r_{p^{+}}^{1}$ with $\theta_{0}$ for different values of cor and $f r$. 


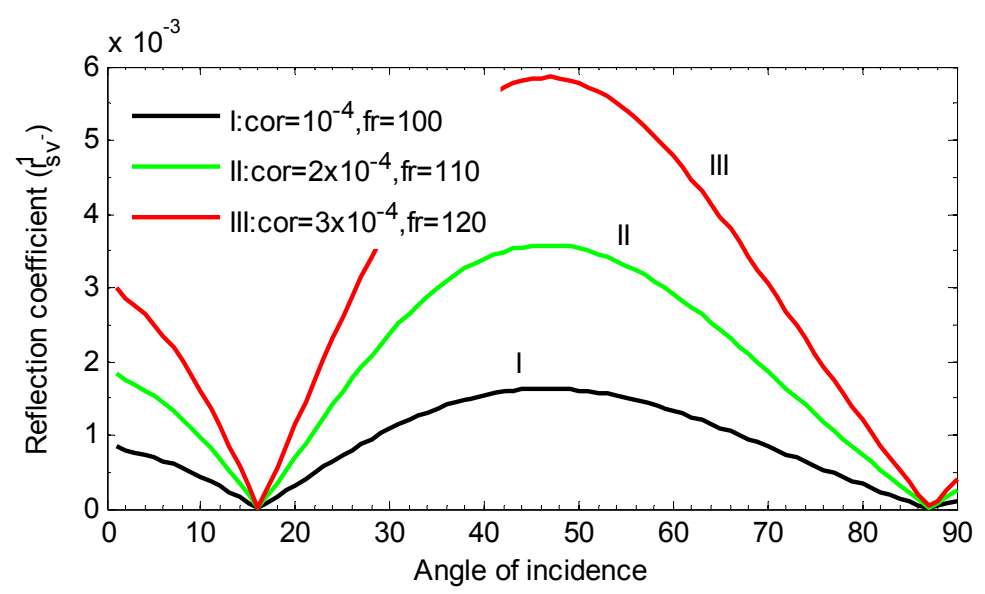

Fig.6 Variation of $r_{s v^{-}}^{l}$ with $\theta_{0}$ for different values of cor and $f r$.

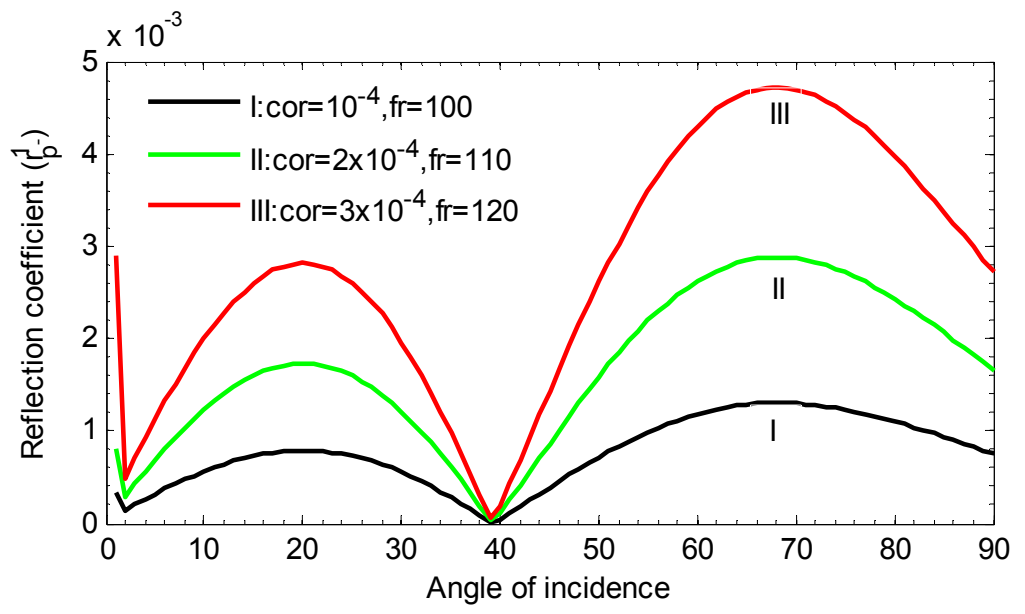

Fig.7. Variation of $r_{p^{-}}^{l}$ with $\theta_{0}$ for different values of cor and $f r$.

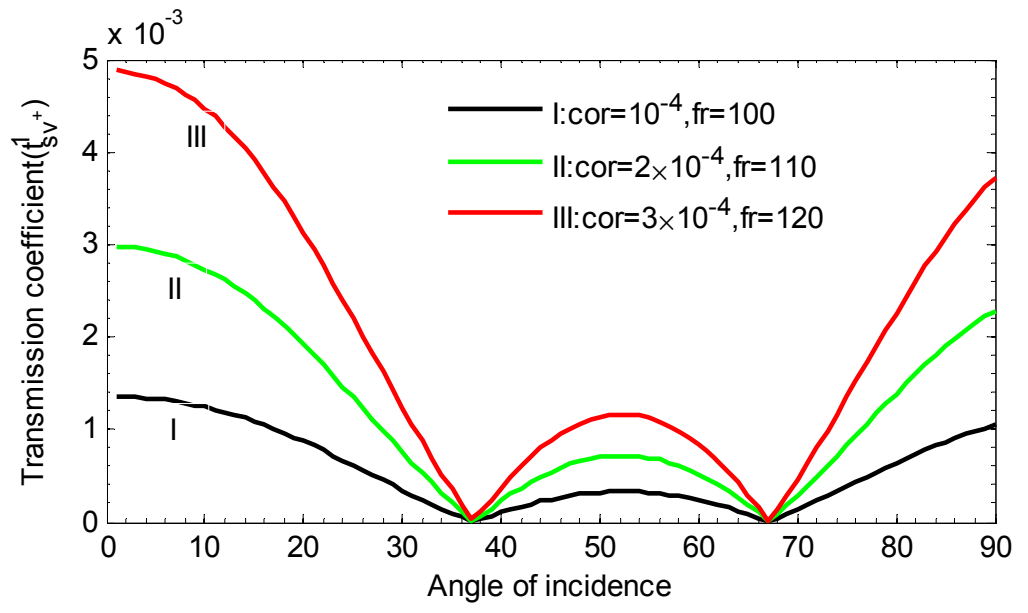

Fig.8. Variation of $t_{s v^{+}}^{1}$ with $\theta_{0}$ for different values of cor and $f r$. 


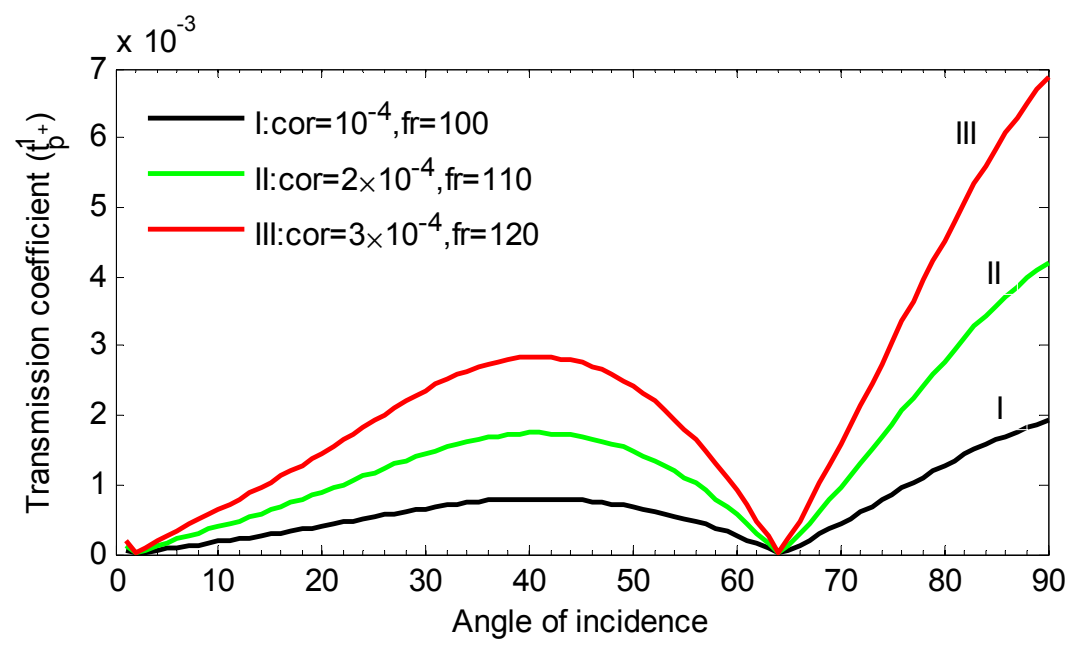

Fig.9. Variation of $t_{p^{+}}^{1}$ with $\theta_{0}$ for different values of cor and $f r$.

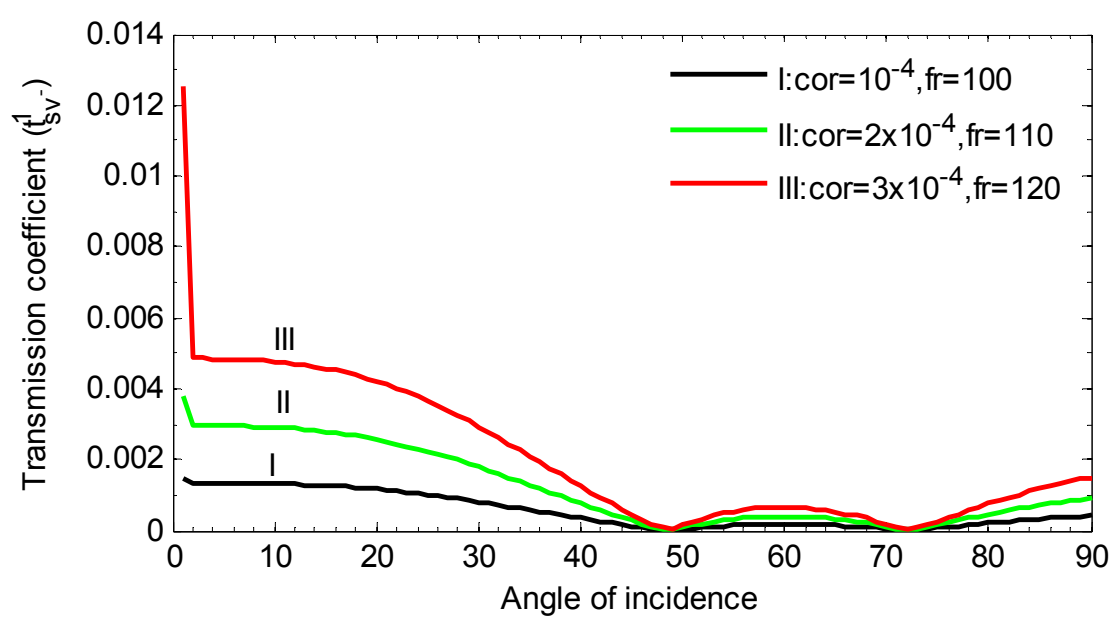

Fig. 10. Variation of $t_{s v^{-}}^{l}$ with $\theta_{0}$ for different values of cor and $f r$.

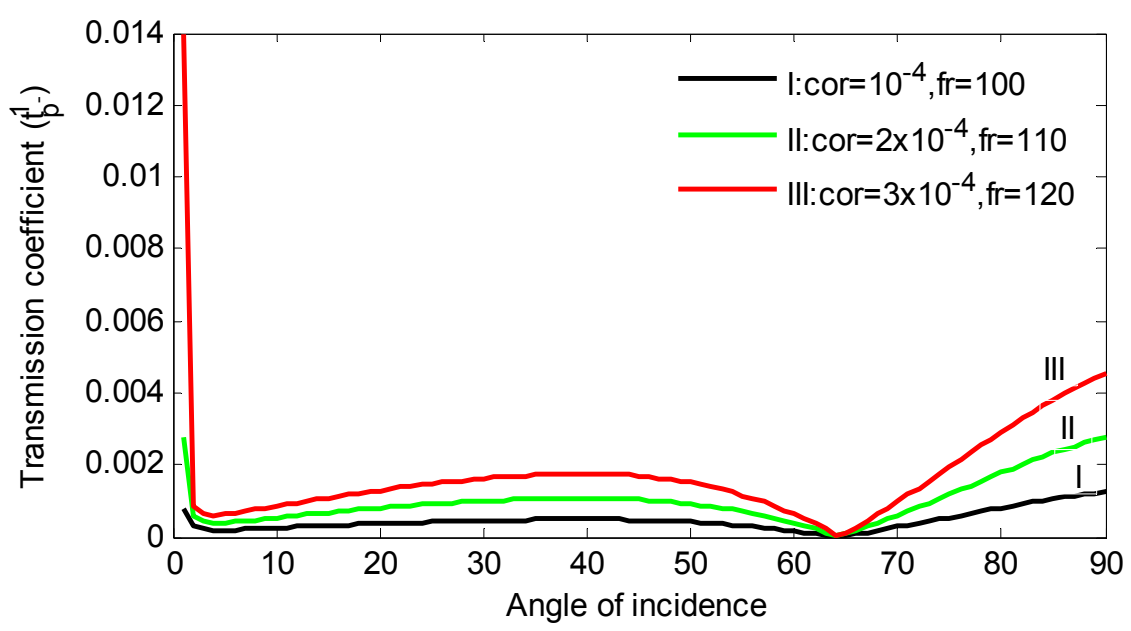

Fig.11. Variation of $t_{p^{-}}^{1}$ with $\theta_{0}$ for different values of cor and $f r$. 
We have seen from Figs 12 and 15 that the coefficients corresponding to the regularly reflected and transmitted waves are independent of corrugation and frequency parameters. In Figs 13, 14, 16 and 17, the coefficients corresponding to the irregularly reflected and transmitted waves are linearly proportional to corrugation and frequency parameters, but at different rates.

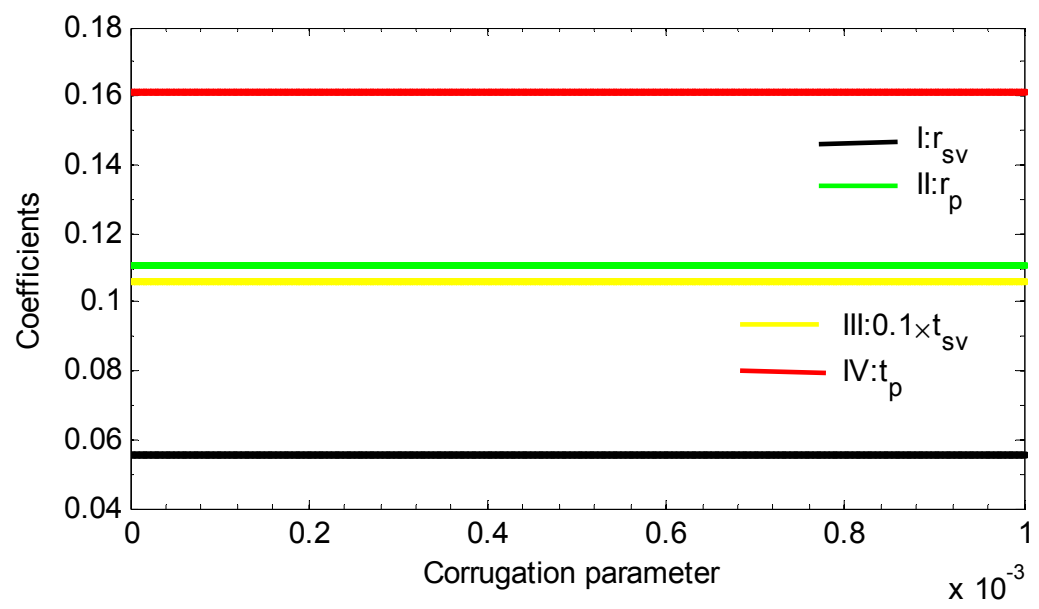

Fig.12. Variation of reflection and transmission coefficients of the regular qSV \& qP-waves with cor.

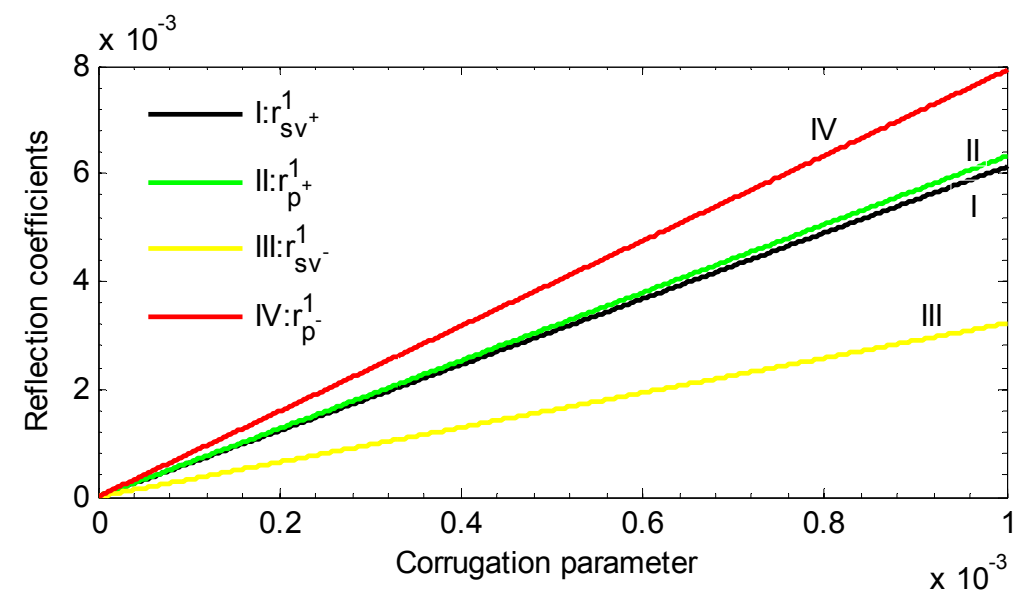

Fig.13. Variation of reflection coefficients of the irregularly reflected qSV \& qP-waves with cor.

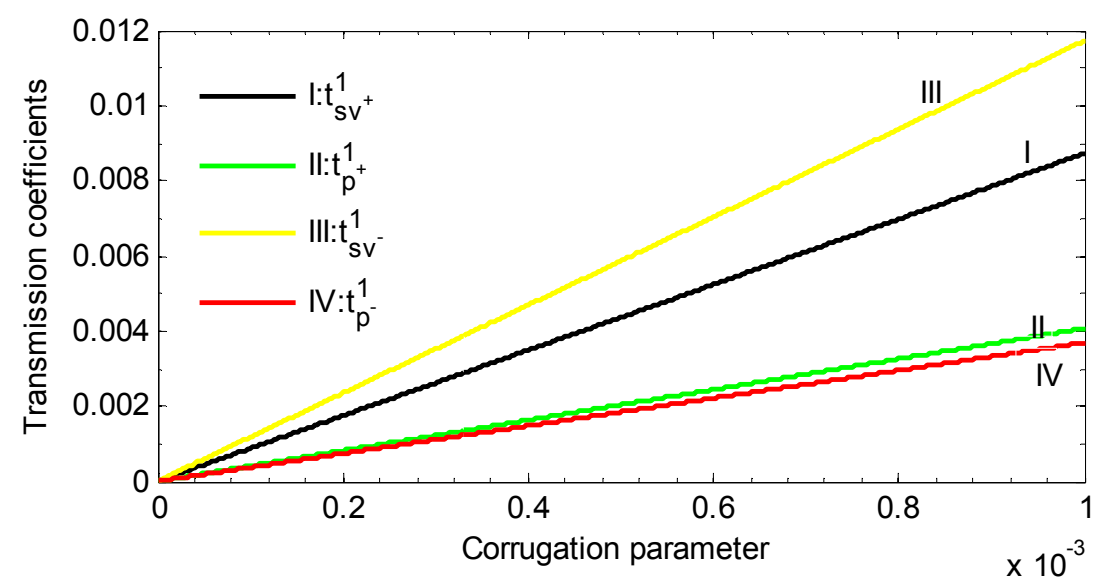

Fig.14. Variation of transmission coefficients of the irregularly transmitted qSV \& qP-waves with cor. 


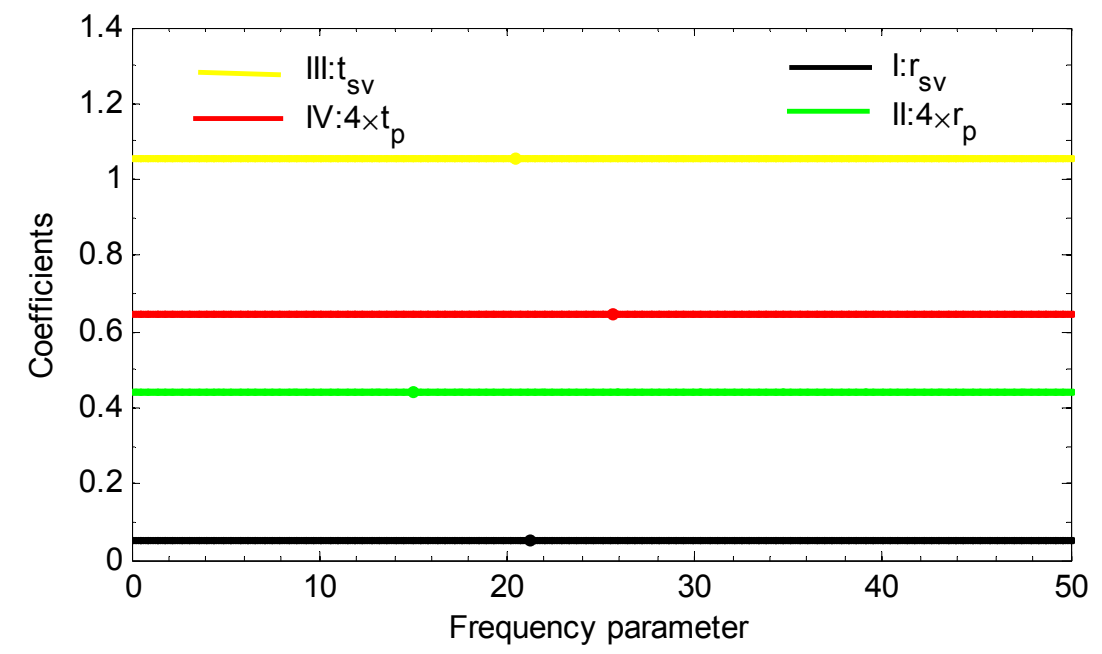

Fig.15. Variation of reflection and transmission coefficients of the regular qSV \& qP-waves with fr.

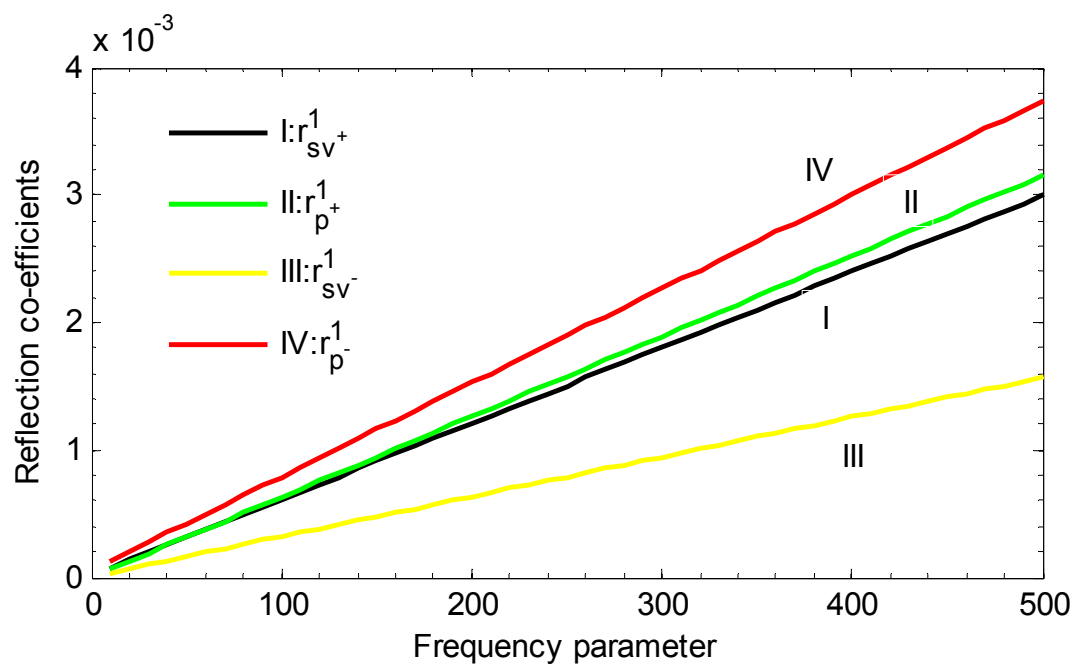

Fig.16. Variation of reflection coefficients of the irregularly reflected qSV \& qP-waves with fr.

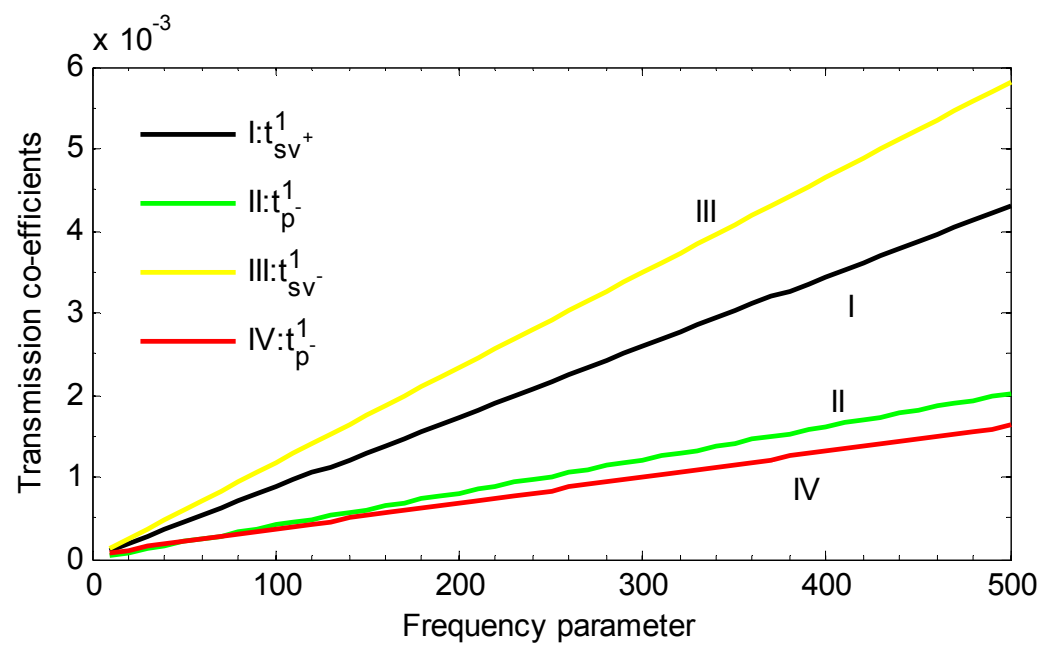

Fig.17. Variation of transmission coefficients of the irregularly transmitted qSV \& qP-waves with fr. 


\section{Conclusion}

The problem of the incident qSV-wave at a corrugated interface between two dissimilar monoclinic elastic half-spaces has been investigated. We have obtained the reflection and transmission coefficients for the first order of approximation corresponding to regularly and irregularly reflected and transmitted qSV and $\mathrm{qP}$-waves with the help of Rayleigh's method of approximation. These coefficients are computed numerically for a specific model and the effect of corrugation and frequency parameters on these coefficients is discussed. We may conclude with the following remarks:

(i) All coefficients corresponding to regular waves are functions of the angle of incidence and elastic constants, while those of irregular waves are found to be functions of the angle of incidence, elastic constants, corrugation and frequency parameters.

(ii) Theoretically and numerically, the reflection and transmission coefficients of the regular waves are independent of corrugation and frequency parameters.

(iii) The coefficients corresponding to irregular waves are found to be linearly proportional to corrugation and frequency parameters.

(iv) It is found that the values of coefficients corresponding to irregular waves increase with an increase of cor and fr.

(v) The values of coefficients corresponding to irregular waves are found to be small.

\section{Nomenclature}

$$
\begin{aligned}
A, D, A_{n}^{ \pm}, D_{n}^{ \pm}, B_{n}^{ \pm}, E_{n}^{ \pm}, G, & - \text { amplitude constants } \\
I, G_{n}^{ \pm}, I_{n}^{ \pm}, H, J, H_{n}^{ \pm}, J_{n}^{ \pm} & \\
c & - \text { phase velocity } \\
c_{i j} & - \text { elastic constant } \\
\boldsymbol{d} & - \text { unit displacement vector } \\
e_{i j} & - \text { strain tensor } \\
F_{0}, F, F_{10}, F_{n}^{ \pm}, F_{I n}^{ \pm}, & - \text {coupling constants } \\
F_{20}, F_{30}, F_{2 n}^{ \pm}, F_{3 n}^{ \pm} & \\
k & - \text { wave number } \\
\boldsymbol{p} & - \text { unit propagation vector } \\
p d & - \text { corrugation parameter } \\
r_{s v}, r_{p}, r_{s v^{ \pm}}^{n}, r_{p^{ \pm}}^{n} & - \text { reflection coefficients } \\
t_{s v}, t_{p}, t_{s v^{ \pm}}^{n}, t_{p^{ \pm}}^{n} & - \text { transmission coefficients } \\
\boldsymbol{u} & - \text { displacement } \\
\zeta_{ \pm n} & - \text { coefficients of Fourier series } \\
\tau_{i j} & - \text { stress tensor } \\
\frac{\omega}{p c_{0}} & - \text { frequency parameter } \\
&
\end{aligned}
$$

\section{References}

[1] Chattopadhyay A. and Choudhury S. (1995): The reflection phenomena of P-waves in a medium of monoclinic type. - Int. J. Engng. Sci., vol.33, No.2, pp.195-207.

[2] Chattopadhyay A., Saha S. and Chakraborty M. (1997): Reflection and transmission of shear waves in monoclinic media. - Int. J. Num. Analy. Methods Geomech., vol.21, No.7, pp.495-504. 
[3] Dowaik M.A. and Ogden R.W. (1991): Interfacial waves and deformations in pre-stressed elastic media. - Proc. Royal Soc. Lon. A, vol.433, pp.313-328.

[4] Sheriff R.E. and Geldart P.L. (1995): Exploration Seismology. - Cambridge: Cambridge University Press.

[5] Singh S.J. (1999): Comments on "The reflection phenomena of P-waves in a medium of monoclinic type by Chattopadhyay A and Choudhury". - Int. J. Engng. Sci., vol.37, No.3, pp. 407-410.

[6] Singh S.J. and Khurana S. (2002): Reflection of $P$ and $S V$-waves at the free surface of a monoclinic elastic halfspace. - Proc. Indian Acad. Sci.(Earth Planet Science), vol.111, No.4, pp.401-412.

[7] Udias A.(1999): Principles of Seismology. - Cambridge: Cambridge University Press.

[8] Chattopadhyay A. and Saha S. (1996): Reflection and refraction of P-waves at the interface of two monoclinic media. - Int. J. Engng. Sci., vol.34, No.11, pp.1271-1284.

[9] Chattopadhyay A. and Saha S. (1999): Reflection and refraction of quasi-SV waves at the interface of the monoclinic media. - Acta Geophys. Pol., vol.47, No.3, pp.307-322. ADDENDUM in Acta Geophys. Pol. (2003), vol.51, No.4, pp.473-475.

[10] Nayfeh A.H. (1991): Elastic wave reflection from liquid-anisotropic substrate interfaces. - Wave Motion, vol.14, No.1, pp.55-67.

[11] Singh S.J. and Khurana S. (2001): Reflection and transmission of P-and SV-waves at the interface between two monoclinic elastic half-spaces. - Proc. Nat. Acad. Sci. A, vol.71, No.4, pp. 305-319.

[12] Rayleigh L. (1907): On the dynamical theory of gratings. - Proc. Royal Soc. Lond. A, vol.79, pp.399-416.

[13] Abubakar I. (1962): Scattering of plane elastic waves at rough surfaces I. - Proc. Camb. Phil. Soc., vol.58, pp.136157.

[14] Asano S. (1966): Reflection and refraction of elastic waves at a corrugated interface. - Bull. Seism. Soc. Am., vol.56, No.1, pp.201-221.

[15] Dunkin J.W. and Eringen A.C. (1962): Reflection of elastic waves from the wavy boundary of a half space. - Int. Proc. $4^{\text {th }}$ US National Cong. Appl. Mech., ASME Publication, pp.143-160.

[16] Gupta S.K. (1978): Reflections and refractions from curved interfaces: model study. - Geophys. Prosp., vol.26, No.1, pp.82-96.

[17] Kuo J.T. and Nafe J.E. (1962): Period equation for Rayleigh waves in a layer overlaying a half space with a sinusoidal interface. - Bull. Seism. Soc. Am., vol. 52, No.4, pp.807-822.

[18] Levy A. and Deresiewicz H. (1967): Reflections and transmission of elastic waves in a system of corrugated layers. - Bull. Seism. Soc. Am., vol.57, No.3, pp.393-419.

[19] Rice O. (1951): Reflection of electromagnetic waves from slightly rough surfaces. - Comm. Pure Appl. Maths., vol.4, No.2-3, pp.351-378.

[20] Singh S.S. and Tomar S.K. (2007): Elastic waves at a corrugated interface between two dissimilar fibre-reinforced elastic half-spaces. - Int. J. Num. Analy. Methods Geomech., vol.31, No.9, pp.1085-1116.

[21] Singh S.S. and Tomar S.K. (2008): qP-wave at a corrugated interface between two dissimilar pre-stressed elastic half-spaces. - J. Sound Vibra., vol.317, No.(3-5), pp.687-708.

[22] Tomar S.K. and Kaur J. (2003): Reflection and transmission of SH-waves at a corrugated interface between two laterally and vertically heterogeneous anisotropic elastic solid half-spaces. - Earth, Planets Space, vol.55, No.9, pp.531-547.

[23] Chattopadhyay A., Gupta S., Sharma V.K. and Kumari P. (2009): Reflection and refraction of plane quasi-P waves at a corrugated interface between distinct triclinic elastic half spaces. - Int. J. Solids Struct., vol.46, No.17, pp.3241-3256.

[24] Kaur J. and Tomar S.K. (2004): Reflection and refraction of SH-waves at a corrugated interface between two monoclinic elastic half-spaces. - Int. J. Num. Analy. Methods Geomech., vol.28, No.15, pp.1543-1575. 
[25] Kennett B.L.N. (1972): Seismic wave scattering by obstacles on interfaces. - Geophys. J. Int., vol.28, No.3, pp.249-266.

[26] Paul A. and Campillo M. (1988): Diffraction and conversion of elastic waves at a corrugated interface. Geophys., vol.53, No.11, pp.1415-1424.

[27] Yu C.W. and Dravinski M. (2009): Scattering of plane harmonic P, SV or Rayleigh waves by a completely embedded corrugated cavity. - Geophys. J. Int., vol.178, No.1, pp.479-487.

[28] Singh S.S. and Singh J. (2013): Effect of corrugation on incident qSV-waves in pre-stressed elastic half-space. Int. J. Appl. Maths. Mech., vol.9, No.9, pp.92-106.

[29] Singh S.S. and Tomar S.K. (2007): Quasi P-waves at a corrugated interface between two dissimilar monoclinic elastic half-spaces. - Int. J. Solids Struct., vol.44, No.1, pp.197-228.

[30] Singh S.S. and Tomar S.K. (2008): Corrigendum to quasi P\$-waves at a corrugated interface between two dissimilar monoclinic elastic half-spaces. - Int. J. Solids Struct., vol.45, No.11, pp.3610-3621.

Received: June 26, 2017

Revised: June 16, 2018 